\title{
VIBRATIONS IN A FLUID-LOADED POROELASTIC HOLLOW CYLINDER SURROUNDED BY A FLUID IN PLANE-STRAIN FORM
}

\author{
B. SHANKER \\ Department of Mathematics, Osmania University \\ Hyderabad - 500 007, INDIA \\ C.N. NATH* \\ Department of Mathematics, CMR Technical Campus \\ Hyderabad - 500 041, INDIA \\ E-mail: nagesh.nath@rediffmail.com \\ S.A. SHAH \\ Department of Mathematics \\ Deccan College of Engineering and Technology \\ Hyderabad - 500 001, INDIA \\ P.M. REDDY \\ Department of Mathematics, Kakatiya University \\ Warangal - 506 009, INDIA
}

\begin{abstract}
Plane-strain vibrations in a fluid-loaded poroelastic hollow cylinder surrounded by a fluid are investigated employing Biot's theory of wave propagation in poroelastic media. The poroelastic hollow cylinder is homogeneous and isotropic, while the inner and outer fluids are homogeneous, isotropic and inviscid. The frequency equation of the fluid-loaded poroelastic cylinder surrounded by a fluid is obtained along with several particular cases, namely, fluid-loaded poroelastic cylinder, fluid-loaded bore, poroelastic cylinder surrounded by a fluid and poroelastic solid cylinder submerged in a fluid. The frequency equations are obtained for axially symmetric, flexural and anti-symmetric vibrations each for a pervious and an impervious surface. Nondimensional frequency for propagating modes is computed as a function of the ratio of thickness to the inner radius of the core. The results are presented graphically for two types of poroelastic cylinders and then discussed.
\end{abstract}

Key words: Biot's theory, fluid-loaded poroelastic cylinder, frequency, submerged poroelastic cylinder, planestrain vibrations, phase velocity.

\section{Introduction}

Plane-strain vibrations in thick-walled hollow elastic cylinders of infinite extent are analyzed by Gazis (1958). Ram Kumar (1966; 1971; 1972) investigated dispersion of various vibrations in empty and fluid-filled hollow cylinders. Sharma and Gogna (1990) studied elastic wave propagation in a cylindrical bore in a liquid saturated poroelastic solid. Plona et al. (1992) studied axially symmetric wave propagation in a fluid-loaded hollow cylinder. Wave propagation in fluid-loaded, transversely isotropic cylinders is studied by Berliner and Solecki (1966). Cui et al. (1997) and Abousleiman and Cui (1998) obtained solutions in an inclined borehole and transversely isotropic well-bore cylinders.

\footnotetext{
${ }^{*}$ To whom correspondence should be addressed
} 
Employing Biot's (1956) theory, Malla Reddy and Tajuddin (2000; 2010) analyzed plane-strain axial symmetric vibrations of a thick-walled hollow poroelastic cylinder and of a poroelastic composite solid cylinder in which they compared the results with those of rule of mixtures. Ahmed Shah (2008) investigated axially symmetric vibrations of fluid-filled poroelastic hollow cylinders in the absence of dissipation. In his investigation, he concluded that the phase velocity of an empty poroelastic hollow cylinder is higher than that of a fluid-filled poroelastic hollow cylinder and the phase velocity of a fluid-filled bore is higher than that of an empty poroelastic bore. Ahmed Shah and Tajuddin (2010) studied flexural vibrations of poroelastic circular hollow cylinders of various wall thicknesses and infinite extent immersed in an acoustic medium. Their study stated that the frequency equation of longitudinal shear vibrations is independent of the nature of surface and the presence of fluid within and around the poroelastic hollow cylinder; also they concluded that there is no significant effect on the phase velocity for a pervious surface with the increase of thickness for the considered materials. They obtained frequency equations of flexural vibrations of a poroelastic solid cylinder as a limiting case. In addition, cutoff frequencies are obtained for both pervious and impervious surfaces when the wavenumber is zero and it is concluded that for zero wavenumber the frequency equation of longitudinal shear vibrations is independent of the nature of the surface. Tajuddin et al. (2011) discussed axial-shear vibrations of an infinitely long poroelastic composite circular cylinder. Nageswara Nath et al. (2011) developed a parametric model of loose bonding between poroelastic halfspaces.

In the present investigation, vibrations in a fluid-loaded poroelastic cylinder surrounded by a fluid are investigated employing Biot's theory of wave propagation in poroelastic media in plane-strain form. The poroelastic hollow cylinder is homogeneous and isotropic, while the inner and outer fluids are homogeneous, isotropic and inviscid. The frequency equation of the fluid-loaded poroelastic cylinder surrounded by a fluid is obtained along with several particular cases, namely, fluid-loaded poroelastic cylinder, fluid-loaded bore, poroelastic cylinder surrounded by a fluid and poroelastic solid cylinder submerged in a fluid. The frequency equations are obtained for axially symmetric, flexural and anti-symmetric vibrations for each pervious and impervious surface. It is observed that shear and dilatational vibrations are uncoupled in the case of axially symmetric vibrations. Non-dimensional frequency for propagating modes is computed as a function of the ratio of thickness to the inner radius of the core for both pervious and impervious surfaces. The results are presented graphically for two types of poroelastic cylinders, namely, sandstone saturated with water and sandstone saturated with kerosene, and then discussed.

\section{Governing equations, formulation and solution of the problem}

The cylindrical polar coordinates are taken as $r, \theta$ and $z$. The model under consideration consists of an infinitely long, homogeneous, isotropic poroelastic hollow cylinder with the inner radius $r_{l}$ and outer radius $r_{2}$, so that the wall thickness of the hollow cylinder is $h\left[=\left(r_{2}-r_{l}\right)\right]$. The axis of the poroelastic hollow cylinder is in the direction of the $z$-axis. The poroelastic hollow cylinder is loaded internally and externally by inviscid fluids. The physical parameters of the two fluids are denoted by a superscript $j(1,2)$ enclosed in parentheses. The parameters with superscript (1) and (2) refer to the inner and outer fluids, respectively. The parameters of the poroelastic hollow cylinder are without any superscript. The geometry of the problem is shown in Fig. 1 and the boundaries of the system are as follows:

For the poroelastic hollow cylinder: $r_{l} \leq r \leq r_{2},-\infty<z<\infty$,

For the inner fluid (1): $\quad 0 \leq r \leq r_{l},-\infty<z<\infty$,

For the outer fluid (2): $\quad r_{2} \leq r<\infty,-\infty<z<\infty$. 


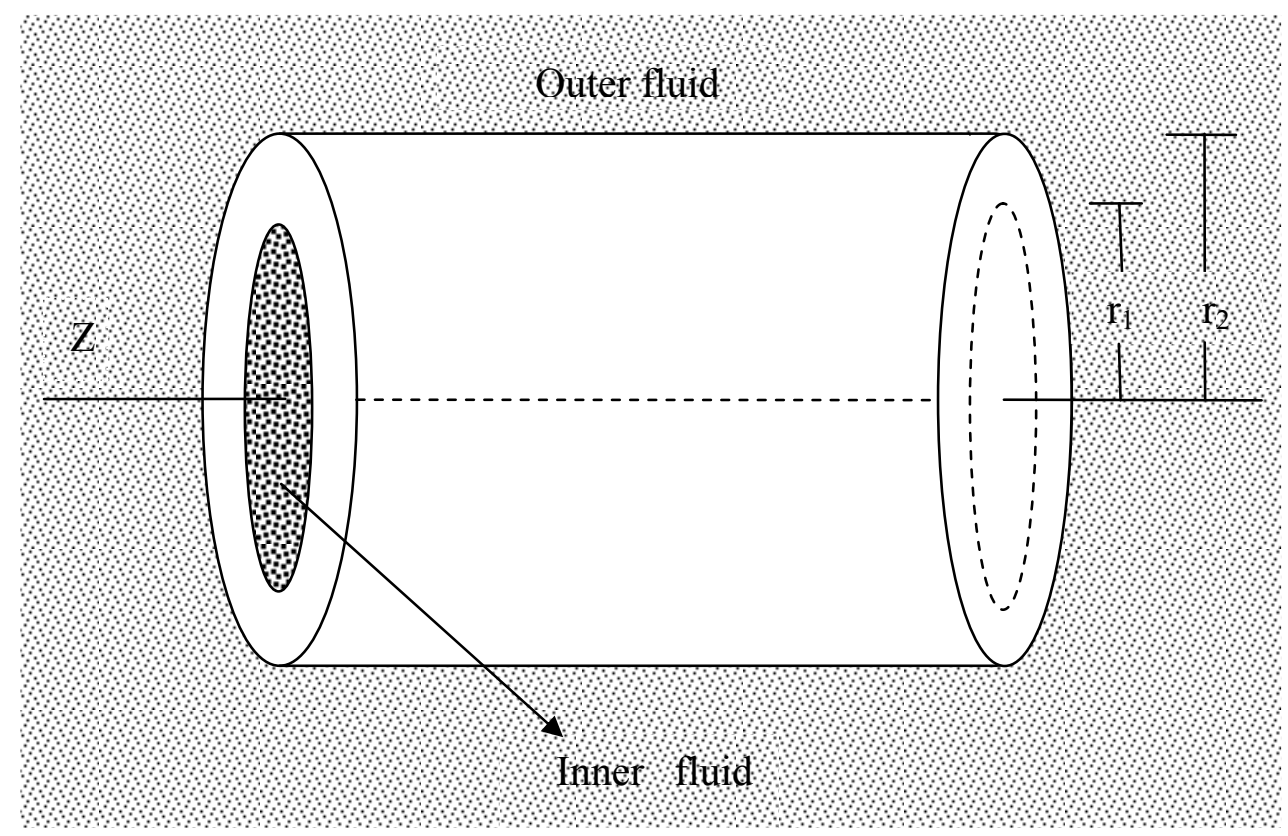

Fig.1 Fluid-loaded poroelastic hollow cylinder surrounded by a fluid.

\subsection{Poroelastic hollow cylinder}

The equations of motion of a homogeneous, isotropic poroelastic solid (Biot, 1956) in the presence of dissipation $b$ are

$$
\begin{aligned}
& N \nabla^{2} \boldsymbol{u}+(A+N) \nabla e+Q \nabla \varepsilon=\frac{\partial^{2}}{\partial t^{2}}\left(\rho_{11} \boldsymbol{u}+\rho_{12} \boldsymbol{U}\right)+b \frac{\partial}{\partial t}(\boldsymbol{u}-\boldsymbol{U}), \\
& Q \nabla e+R \nabla \varepsilon=\frac{\partial^{2}}{\partial t^{2}}\left(\rho_{12} \boldsymbol{u}+\rho_{22} \boldsymbol{U}\right)-b \frac{\partial}{\partial t}(\boldsymbol{u}-\boldsymbol{U})
\end{aligned}
$$

where $\nabla^{2}$ is the Laplacian operator, $\boldsymbol{u}(u, v, w)$ and $\boldsymbol{U}(U, V, W)$ are displacements of the solid and liquid media, respectively, while $e$ and $\varepsilon$ are dilatations of the solid and liquid, respectively; $A, N, Q, R$ are all poroelastic constants and $\rho_{11}, \rho_{12}, \rho_{22}$ are the mass coefficients following Biot (1956). The poroelastic constants $A$ and $N$ correspond to familiar Lam'e constants in a purely elastic solid. The coefficient $N$ represents the shear modulus of the solid. The coefficient $R$ is a measure of the pressure required on the liquid to force a certain amount of the liquid into the aggregate while the total volume remains constant. The coefficient $Q$ represents the coupling between the volume changes of solid to that of liquid. The stresses $\sigma_{k l}$ and the liquid pressure $s$ of the poroelastic solid are

$$
\begin{aligned}
& \sigma_{k l}=2 N e_{k l}+(A e+Q \varepsilon) \delta_{k l}, \quad(k, l=r, \theta, z), \\
& s=Q e+R \varepsilon,
\end{aligned}
$$

where $\delta_{k l}$ is the well-known Kronecker delta function.

The displacements of solid $\boldsymbol{u}(u, v, w)$, which can readily be evaluated from field Eq.(2.1) are 


$$
\begin{aligned}
& u=\left[C_{1} \xi_{1} J_{n}^{\prime}\left(\xi_{1} r\right)+C_{2} \xi_{1} Y_{n}^{\prime}\left(\xi_{1} r\right)+C_{3} \xi_{2} J_{n}^{\prime}\left(\xi_{2} r\right)+\right. \\
& \left.+C_{4} \xi_{2} Y_{n}^{\prime}\left(\xi_{2} r\right)+A_{1} \frac{n}{r} J_{n}\left(\xi_{3} r\right)+B_{1} \frac{n}{r} Y_{n}\left(\xi_{3} r\right)\right] \cos n \theta e^{i \omega t}, \\
& v=-\left[C_{1} \frac{n}{r} J_{n}\left(\xi_{1} r\right)+C_{2} \frac{n}{r} Y_{n}\left(\xi_{1} r\right)+C_{3} \frac{n}{r} J_{n}\left(\xi_{2} r\right)+\right. \\
& \left.+C_{4} \frac{n}{r} Y_{n}\left(\xi_{2} r\right)+A_{1} \xi_{3} J_{n}^{\prime}\left(\xi_{3} r\right)+B_{1} \xi_{3} Y_{n}^{\prime}\left(\xi_{3} r\right)\right] \sin n \theta e^{i \omega t}
\end{aligned}
$$

where $C_{1}, C_{2}, C_{3}, C_{4}, A_{1}$, and $B_{1}$ are constants, $\omega$ is the circular frequency, $n$ is the angular wavenumber, $\xi_{i}=\frac{\omega}{V_{i}}(i=1,2,3) ; V_{1}, V_{2}$ are dilatational wave velocities of the first and second kind, respectively, and $V_{3}$ is the shear wave velocity.

By substituting the displacements solutions from Eq.(2.3) into the Eq.(2.2), the relevant stresses are

$$
\begin{aligned}
& \sigma_{r r}+s=\left\{C_{1} \xi_{1}^{2}\left[2 N J_{n}^{\prime \prime}\left(\xi_{1} r\right)+\left[(Q+R) \delta_{1}^{2}-(A+Q)\right] J_{n}\left(\xi_{1} r\right)\right]+\right. \\
& +C_{2} \xi_{1}^{2}\left[2 N Y_{n}^{\prime \prime}\left(\xi_{1} r\right)+\left[(Q+R) \delta_{1}^{2}-(A+Q)\right] Y_{n}\left(\xi_{1} r\right)\right]+ \\
& +C_{3} \xi_{2}^{2}\left[2 N J_{n}^{\prime \prime}\left(\xi_{2} r\right)+\left[(Q+R) \delta_{2}^{2}-(A+Q)\right] J_{n}\left(\xi_{2} r\right)\right]+ \\
& +C_{4} \xi_{2}^{2}\left[2 N Y_{n}^{\prime \prime}\left(\xi_{2} r\right)+\left[(Q+R) \delta_{2}^{2}-(A+Q)\right] Y_{n}\left(\xi_{2} r\right)\right]+ \\
& \left.+A_{1} \frac{n}{r}\left[\xi_{3} J_{n}^{\prime}\left(\xi_{3} r\right)-\frac{1}{r} J_{n}\left(\xi_{3} r\right)\right]+B_{1} \frac{n}{r}\left[\xi_{3} Y_{n}^{\prime}\left(\xi_{3} r\right)-\frac{1}{r} Y_{n}\left(\xi_{3} r\right)\right]\right\} \cos n \theta e^{i \omega t}, \\
& \sigma_{r \theta}=\left\{C_{1}\left[\frac{n N}{r^{2}} J_{n}\left(\xi_{1} r\right)-\frac{2 n N}{r} \xi_{1} J_{n}^{\prime}\left(\xi_{1} r\right)\right]+C_{2}\left[\frac{n N}{r^{2}} Y_{n}\left(\xi_{1} r\right)-\frac{2 n N}{r} \xi_{1} Y_{n}^{\prime}\left(\xi_{1} r\right)\right]+\right. \\
& +C_{3}\left[\frac{n N}{r^{2}} J_{n}\left(\xi_{2} r\right)-\frac{2 n N}{r} \xi_{2} J_{n}^{\prime}\left(\xi_{2} r\right)\right]+C_{4}\left[\frac{n N}{r^{2}} Y_{n}\left(\xi_{2} r\right)-\frac{2 n N}{r} \xi_{2} Y_{n}^{\prime}\left(\xi_{2} r\right)\right]+ \\
& -A_{1}\left[\xi_{3}^{2} J_{n}^{\prime \prime}\left(\xi_{3} r\right)-\frac{\xi_{3}}{r} J_{n}^{\prime}\left(\xi_{3} r\right)+\frac{n^{2}}{r^{2}} J_{n}\left(\xi_{3} r\right)\right]+ \\
& \left.-B_{1}\left[\xi_{3}^{2} Y_{n}^{\prime \prime}\left(\xi_{3} r\right)-\frac{\xi_{3}}{r} Y_{n}^{\prime}\left(\xi_{3} r\right)+\frac{n^{2}}{r^{2}} Y_{n}\left(\xi_{3} r\right)\right]\right\} \sin n \theta e^{i \omega t}, \\
& s=\left\{C_{1} \xi_{1}^{2}\left(R \delta_{1}^{2}-Q\right) J_{n}\left(\xi_{1} r\right)+C_{2} \xi_{1}^{2}\left(R \delta_{1}^{2}-Q\right) Y_{n}\left(\xi_{1} r\right)+\right. \\
& \left.+C_{3} \xi_{2}^{2}\left(R \delta_{2}^{2}-Q\right) J_{n}\left(\xi_{2} r\right)+C_{4} \xi_{2}^{2}\left(R \delta_{2}^{2}-Q\right) Y_{n}\left(\xi_{2} r\right)\right\} \cos n \theta e^{i \omega t}, \\
& \frac{\partial s}{\partial r}=\left\{C_{1} \xi_{1}^{3}\left(R \delta_{1}^{2}-Q\right) J_{n}^{\prime}\left(\xi_{1} r\right)+C_{2} \xi_{1}^{3}\left(R \delta_{1}^{2}-Q\right) Y_{n}^{\prime}\left(\xi_{1} r\right)+\right. \\
& \left.+C_{3} \xi_{2}^{3}\left(R \delta_{2}^{2}-Q\right) J_{n}^{\prime}\left(\xi_{2} r\right)+C_{4} \xi_{2}^{2}\left(R \delta_{2}^{2}-Q\right) Y_{n}^{\prime}\left(\xi_{2} r\right)\right\} \cos n \theta e^{i \omega t} . \\
&
\end{aligned}
$$

In Eq.(2.4),

$$
\delta_{k}^{2}=\left[\left(\mathrm{RM}_{11}-Q \mathrm{M}_{12}\right)-V_{k}^{-1}\left(P R-Q^{2}\right)\right]\left(\mathrm{RM}_{12}-Q \mathrm{M}_{22}\right)^{-1}, \quad \text { for } \quad k=1,2
$$


where $P=A+2 N$ is a poroelastic constant and $M_{11}=\rho_{11}-i b \omega^{-1}$,

$$
M_{12}=\rho_{12}+i b \omega^{-1} \text { and } \quad M_{22}=\rho_{22}-i b \omega^{-1}
$$

\subsection{Inviscid elastic fluid}

The equation of motion for a homogeneous, isotropic, inviscid elastic fluid is

$$
\nabla^{2} \Phi^{(j)}=\frac{1}{V_{f}^{(j)^{2}}} \frac{\partial^{2} \Phi^{(j)}}{\partial^{2} t}, \quad(j=1,2)
$$

where $\Phi^{(j)}$ is the displacement potential function and $V_{f}^{(j)}$ is the velocity of sound in fluid.

The fluid pressure $P_{f}^{(j)}$ is given by

$$
P_{f}^{(j)}=-\rho_{f}^{(j)} \frac{\partial^{2} \Phi^{(j)}}{\partial^{2} t}
$$

where $\rho_{f}^{(j)}$ is the density of the fluid. The superscripts $j_{=} 1,2$ represent the inner and outer fluids, respectively.

Using the displacement potential function, the displacement $\boldsymbol{u}_{f}^{(I)}=\left(u_{f}^{(I)}, v_{f}^{(I)}, 0\right)$ and fluid pressure $P_{f}^{(l)}$ of the inner fluid are obtained as

$$
\begin{aligned}
& u_{f}^{(I)}=A_{f}^{(I)} \xi_{f}^{(I)} J_{n}^{\prime}\left(\xi_{f}^{(I)} r\right) \cos n \theta e^{i \omega t}, \\
& v_{f}^{(I)}=-A_{f}^{(I)} \frac{1}{r} J_{n}\left(\xi_{f}^{(I)} r\right) \sin n \theta e^{i \omega t}, \\
& p_{f}^{(I)}=A_{f}^{(I)} \rho_{f}^{(I)} \omega^{2} J_{n}\left(\xi_{f}^{(l)} r\right) \cos n \theta e^{i \omega t} .
\end{aligned}
$$

Similarly, the displacement $\boldsymbol{u}_{f}^{(2)}=\left(u_{f}^{(2)}, v_{f}^{(2)}, 0\right)$ and fluid pressure $P_{f}^{(2)}$ of the outer fluid are

$$
\begin{aligned}
& u_{f}^{(2)}=A_{f}^{(2)} \xi_{f}^{(2)} H_{n}^{(1)^{\prime}}\left(\xi_{f}^{(2)} r\right) \cos n \theta e^{i \omega t}, \\
& v_{f}^{(2)}=-A_{f}^{(2)} \frac{1}{r} H_{n}^{(1)}\left(\xi_{f}^{(2)} r\right) \sin n \theta e^{i \omega t}, \\
& p_{f}^{(2)}=A_{f}^{(2)} \rho_{f}^{(2)} \omega^{2} H_{n}^{(I)}\left(\xi_{f}^{(2)} r\right) \cos n \theta e^{i \omega t} .
\end{aligned}
$$

In Eqs (2.7) and (2.8), $\xi_{f}^{(j)}=\frac{\omega}{V_{f}^{(j)}}, A_{f}^{(j)}$ is a constant and $H_{n}^{(l)}$ is the Hankel function of first kind and of order $n$. 


\section{Boundary conditions and frequency equation}

For a perfect contact between the poroelastic hollow cylinder and the fluids, we assume that the normal stresses and displacements are continuous at $r=r_{1}$ and $r=r_{2}$. Thus, the boundary conditions in the case of a pervious surface are

$$
\begin{array}{lllll}
\sigma_{r r}+s=-p_{f}^{(1)}, & \sigma_{r \theta}=0, & u=u_{f}^{(1)}, & s=p_{f}^{(1)}, \quad \text { at } & r=r_{1}, \\
\sigma_{r r}+s=-p_{f}^{(2)}, & \sigma_{r \theta}=0, & u=u_{f}^{(2)}, & s=p_{f}^{(2)}, \quad \text { at } & r=r_{2} .
\end{array}
$$

The boundary conditions in the case of an impervious surface are

$$
\begin{array}{llll}
\sigma_{r r}+s=-p_{f}^{(l)}, & \sigma_{r \theta}=0, & u=u_{f}^{(1)}, & \frac{\partial s}{\partial r}=\frac{\partial p_{f}^{(1)}}{\partial r}, \quad \text { at } \quad r=r_{l}, \\
\sigma_{r r}+s=-p_{f}^{(2)}, & \sigma_{r \theta}=0, & u=u_{f}^{(2)}, & \frac{\partial s}{\partial r}=\frac{\partial p_{f}^{(2)}}{\partial r}, \quad \text { at } \quad r=r_{2} .
\end{array}
$$

Equations (3.1) result in a system of eight homogeneous equations with eight constants $C_{1}, C_{2}, C_{3}, C_{4}, A_{1}, B_{1}, A_{f}^{(1)}$ and $A_{f}^{(2)}$. For a nontrivial solution, the determinant of the coefficients must vanish. By eliminating these constants, the frequency equation of vibrations of the fluid-loaded poroelastic hollow cylinder surrounded by a fluid for a pervious surface is

$$
\left|C_{i j}\right|=0, \quad i, j=1,2,3 \ldots 8
$$

where the elements $C_{i j}$ are given by

$$
\begin{aligned}
& C_{11}=\xi_{1}^{2} r_{l}^{2}\left[2 N J_{n}^{\prime \prime}\left(\xi_{1} r_{l}\right)+\left[(Q+R) \delta_{1}^{2}-(A+Q)\right] J_{n}\left(\xi_{1} r_{l}\right)\right], \\
& C_{15}=n\left[\xi_{3} r_{l} J_{n}^{\prime}\left(\xi_{3} r_{1}\right)-J_{n}\left(\xi_{3} r_{1}\right)\right], C_{17}=\rho_{f}^{(I)} \omega^{2} r_{1}^{2} J_{n}\left(\xi_{f}^{(1)} r_{1}\right), \\
& C_{21}=\left[2 n N J_{n}\left(\xi_{1} r_{1}\right)-2 n N \xi_{1} r_{l} J_{n}^{\prime}\left(\xi_{1} r_{1}\right)\right], \\
& C_{25}=-\left[\xi_{3}^{2} r_{1}^{2} J_{n}^{\prime \prime}\left(\xi_{3} r_{l}\right)-\xi_{3} r_{l} J_{n}^{\prime}\left(\xi_{3} r_{l}\right)+n^{2} J_{n}\left(\xi_{3} r_{l}\right)\right], \\
& C_{31}=\xi_{1} r_{l} J_{n}^{\prime}\left(\xi_{1} r_{1}\right), \quad C_{35}=n J_{n}\left(\xi_{1} r_{1}\right), \quad C_{37}=-\xi_{f}^{(l)} r_{l} J_{n}^{\prime}\left(\xi_{f}^{(I)} r_{l}\right), \\
& C_{41}=\xi_{1}^{2} r_{1}^{2}\left(R \delta_{1}^{2}-Q\right) J_{n}\left(\xi_{1} r_{1}\right), \quad C_{47}=C_{17},
\end{aligned}
$$

$C_{i 3}=C_{i 1}$ for $i=1,2,3,4$ with replacing $J_{n}$ and its derivatives, respectively, by $Y_{n}$ and its derivatives $C_{i 2}=C_{i 1} ; C_{i 4}=C_{i 2} ; C_{i 6}=C_{i 5}$ for $i=1,2,3,4$ with replacing $\xi_{1}, \delta_{1}$, respectively, by $\xi_{2}, \delta_{2}$, 


$$
\begin{aligned}
& C_{18}=C_{27}=C_{28}=C_{38}=C_{45}=C_{46}=C_{48}=0, \\
& C_{5 j}=C_{1 j}, C_{6 j}=C_{2 j}, C_{7 j}=C_{3 j}, C_{8 j}=C_{4 j} \text { for } j=1,2, \ldots, 6 \text { with replacing } r_{1} \text { by } r_{2}, \\
& C_{58}=\rho_{f}^{(2)} \omega^{2} r_{2}^{2} H_{n}^{(1)}\left(\xi_{f}^{(2)} r_{2}\right), \quad C_{68}=0, \\
& C_{78}=-\xi_{f}^{(2)} r_{2} H_{n}^{(I) \prime}\left(\xi_{f}^{(2)} r_{2}\right), \\
& C_{88}=C_{58}, \\
& C_{i 7}=0, \text { for } j=5,6,7,8 .
\end{aligned}
$$

Proceeding on similar lines, by employing the boundary conditions (3.2), the frequency equation of vibrations of the fluid-loaded poroelastic hollow cylinder surrounded by a fluid for an impervious surface is obtained as

$$
\left|D_{i j}\right|=0, \quad i, j=1,2,3 \ldots 8
$$

where the elements $D_{i j}$ are

$$
\begin{aligned}
& D_{i j}=C_{i j} \quad \text { for } \quad i=1,2,3,5,6,7 ; \quad j=1,2, \ldots 8, \\
& D_{41}=\xi_{1}^{3} r_{1}^{3}\left(R \delta_{1}^{2}-Q\right) J_{n}^{\prime}\left(\xi_{1} r_{1}\right), \quad D_{43}=\xi_{2}^{3} r_{1}^{3}\left(R \delta_{2}^{2}-Q\right) J_{n}^{\prime}\left(\xi_{2} r_{1}\right), \\
& D_{42}=D_{41} ; D_{44}=D_{43} \text { with replacing } J_{n} \text { and its derivatives, respectively, } \\
& \text { by } Y_{n} \text { and its derivatives, } \\
& D_{45}=D_{46}=D_{48}=0, \quad D_{47}=\rho_{f}^{(1)} \omega^{2} r_{1}^{3} \xi_{f}^{(1)} J_{n}^{\prime}\left(\xi_{f}^{(1)} r_{1}\right), \\
& D_{8 j}=D_{4 j} \quad \text { for } \quad j=1,2,3,4, \quad \text { with replacing } r_{2} \text { by } r_{1}, \\
& D_{85}=D_{86}=D_{87}=0, \quad D_{88}=\rho_{f}^{(2)} \omega^{2} r_{2}^{3} \xi_{f}^{(2)} H_{n}^{(2)^{\prime}}\left(\xi_{f}^{(2)} r_{2}\right),
\end{aligned}
$$

\section{Axially symmetric vibrations $(n=0)$}

For axially symmetric vibrations, the frequency Eq.(3.3) of vibrations of the fluid-loaded poroelastic hollow cylinder surrounded by a fluid for a pervious surface reduces to

$$
A_{1} A_{2}=0,
$$

with

$$
A_{1}=\left|\begin{array}{ll}
C_{25} & C_{26} \\
C_{65} & C_{66}
\end{array}\right|, \quad A_{2}=\left|\begin{array}{llllll}
C_{11} & C_{12} & C_{13} & C_{14} & C_{17} & 0 \\
C_{31} & C_{32} & C_{33} & C_{34} & C_{37} & 0 \\
C_{41} & C_{42} & C_{43} & C_{44} & C_{47} & 0 \\
C_{51} & C_{52} & C_{53} & C_{54} & 0 & C_{58} \\
C_{71} & C_{72} & C_{73} & C_{74} & 0 & C_{78} \\
C_{81} & C_{82} & C_{83} & C_{84} & 0 & C_{88}
\end{array}\right|,
$$

where the elements $C_{i j}$ are defined in Eq.(3.4).

From Eq.(3.7) we obtain $A_{1}=0$ or $A_{2}=0$. On simplification, the equation

$$
A_{1}=0,
$$

reduces to

$$
J_{2}\left(\xi_{3} r_{1}\right) Y_{2}\left(\xi_{3} r_{2}\right)-J_{2}\left(\xi_{3} r_{2}\right) Y_{2}\left(\xi_{3} r_{1}\right)=0 \text {, }
$$


which is independent of fluid parameters and dilatational vibrations, hence this is the frequency equation of axially symmetric shear vibrations of the poroelastic hollow cylinder for a pervious surface obtained by Malla Reddy and Tajuddin (2000).

The equation

$$
A_{2}=0,
$$

is the frequency equation of dilatational vibrations of the fluid-loaded poroelastic hollow cylinder surrounded by a fluid for a pervious surface. Equation (3.7) shows that the axially symmetric shear and dilatational vibrations of the fluid-loaded poroelastic hollow cylinder surrounded by a fluid for a pervious surface are uncoupled.

Similarly, the frequency Eq.(3.5) of vibrations of the fluid-loaded poroelastic hollow cylinder surrounded by a fluid for an impervious surface reduces to

$$
B_{1} B_{2}=0,
$$

with $\quad B_{1}=\left|\begin{array}{cc}D_{25} & D_{26} \\ D_{65} & D_{66}\end{array}\right|, \quad B_{2}=\left|\begin{array}{cccccc}D_{31} & D_{32} & D_{33} & D_{34} & D_{37} & 0 \\ D_{41} & D_{42} & D_{43} & D_{44} & D_{47} & 0 \\ D_{51} & D_{52} & D_{53} & D_{54} & 0 & D_{58} \\ D_{71} & D_{72} & D_{73} & D_{74} & 0 & D_{78} \\ D_{81} & D_{82} & D_{83} & D_{84} & 0 & D_{88}\end{array}\right|$,

where the elements $D_{i j}$ are defined in Eq.(3.6).

From Eq.(3.11), it is clear that $B_{1}=0$ or $B_{2}=0$. On simplification, the equation

$$
B_{1}=0 \text {, }
$$

reduces to

$$
J_{2}\left(\xi_{3} r_{1}\right) Y_{2}\left(\xi_{3} r_{2}\right)-J_{2}\left(\xi_{3} r_{2}\right) Y_{2}\left(\xi_{3} r_{1}\right)=0,
$$

which is same as Eq.(3.9a).

The equation

$$
B_{2}=0,
$$

is the frequency equation of axially symmetric dilatational vibrations of the fluid-loaded poroelastic hollow cylinder surrounded by a fluid for an impervious surface. Equation (4.11) shows that the axially symmetric shear and dilatational vibrations of the fluid-loaded poroelastic hollow cylinder surrounded by a fluid for an impervious surface are uncoupled.

From Eqs (3.9a) and (3.13a), it is clear that the frequency equation of axially symmetric shear vibrations of the fluid-loaded poroelastic hollow cylinder surrounded by a fluid is same for a pervious and an impervious surface. Hence, axially symmetric shear vibrations of the fluid-loaded poroelastic hollow cylinder surrounded by a fluid are independent of the nature of the surface.

\section{Particular cases}

Under suitable conditions the fluid-loaded poroelastic hollow cylinder surrounded by a fluid reduces to the following particular cases

4.1. Fluid-loaded poroelastic hollow cylinder,

4.1.1. Fluid-loaded poroelastic bore,

4.1.1a. Poroelastic bore,

4.2. Poroelastic hollow cylinder surrounded by a fluid,

4.2.1. Poroelastic solid cylinder submerged in a fluid. 


\subsection{Fluid-loaded poroelastic hollow cylinder}

When the fluid density $\rho_{f}^{(2)}$ of the outer fluid is zero, then the fluid-loaded poroelastic hollow cylinder surrounded by a fluid reduces to the fluid-loaded poroelastic hollow cylinder. In this case, the frequency Eq.(3.3) of the fluid-loaded poroelastic hollow cylinder surrounded by a fluid for a pervious surface reduces to

$$
\left|\begin{array}{lllllll}
C_{11} & C_{12} & C_{13} & C_{14} & C_{15} & C_{16} & C_{17} \\
C_{21} & C_{22} & C_{23} & C_{24} & C_{25} & C_{26} & 0 \\
C_{31} & C_{32} & C_{33} & C_{34} & C_{35} & C_{36} & C_{37} \\
C_{41} & C_{42} & C_{43} & C_{44} & 0 & 0 & C_{47} \\
C_{51} & C_{52} & C_{53} & C_{54} & C_{55} & C_{56} & 0 \\
C_{61} & C_{62} & C_{63} & C_{64} & C_{65} & C_{66} & 0 \\
C_{81} & C_{82} & C_{83} & C_{84} & 0 & 0 & 0
\end{array}\right|=0,
$$

where the elements $C_{i j}$ are defined in Eq.(3.4).

This is the frequency equation of the fluid-loaded poroelastic hollow cylinder for a pervious surface.

In a similar way, the frequency Eq.(3.5) of the fluid-loaded poroelastic hollow cylinder surrounded by fluid for an impervious surface reduces to

$$
\left|\begin{array}{lllllll}
D_{11} & D_{12} & D_{13} & D_{14} & D_{15} & D_{16} & D_{17} \\
D_{21} & D_{22} & D_{23} & D_{24} & D_{25} & D_{26} & 0 \\
D_{31} & D_{32} & D_{33} & D_{34} & D_{35} & D_{36} & D_{37} \\
D_{41} & D_{42} & D_{43} & D_{44} & 0 & 0 & D_{47} \\
D_{51} & D_{52} & D_{53} & D_{54} & D_{55} & D_{56} & 0 \\
D_{61} & D_{62} & D_{63} & D_{64} & D_{65} & D_{66} & 0 \\
D_{81} & D_{82} & D_{83} & D_{84} & 0 & 0 & 0
\end{array}\right|=0
$$

where the elements $D_{i j}$ are defined in Eq.(3.6).

This is the frequency equation of the fluid-loaded poroelastic hollow cylinder for an impervious surface.

\section{Axially symmetric vibrations $(n=0)$}

For axially symmetric vibrations, the frequency Eq.(4.1) of vibrations of the fluid-loaded poroelastic hollow cylindrical for a pervious surface reduces to

$$
\begin{aligned}
& E_{1} E_{2}=0, \\
& E_{1}=\left|\begin{array}{ll}
C_{25} & C_{26} \\
C_{65} & C_{66}
\end{array}\right|, \quad E_{2}=\left|\begin{array}{lllll}
C_{11} & C_{12} & C_{13} & C_{14} & C_{17} \\
C_{31} & C_{32} & C_{33} & C_{34} & C_{37} \\
C_{41} & C_{42} & C_{43} & C_{44} & C_{47} \\
C_{51} & C_{52} & C_{53} & C_{54} & 0 \\
C_{81} & C_{82} & C_{83} & C_{84} & 0
\end{array}\right|,
\end{aligned}
$$

where the elements $C_{i j}$ are defined in Eq.(3.4).

From Eq.(4.3) we obtain $E_{1}=0$ or $E_{2}=0$. In particular, the equation

$$
E_{l}=0
$$

is the frequency equation of axially symmetric shear vibrations of the poroelastic hollow cylinder for a pervious surface and is same as the equation $A_{1}=0$ obtained in Eq.(3.9). 
The equation

$$
E_{2}=0 \text {, }
$$

is the frequency equation of axially symmetric dilatational vibrations of the fluid-loaded poroelastic hollow cylinder for a pervious surface. Equation (4.3) shows that the shear and dilatational vibrations of the fluidloaded poroelastic hollow cylinder for a pervious surface are uncoupled.

Similarly, the frequency Eq.(4.2) of vibrations of the fluid-loaded poroelastic hollow cylinder for an impervious surface reduces to

with

$$
E_{3} E_{4}=0,
$$

where the elements $D_{i j}$ are defined in Eq.(3.6).

From Eq.(4.7), it is clear that $E_{3}=0$ or $E_{4}=0$. In particular, the equation

$$
E_{3}=0 \text {, }
$$

is the frequency equation of axially symmetric shear vibrations of the fluid-loaded poroelastic hollow cylinder for an impervious surface and is same as the equation $B_{I}=0$ obtained in Eq.(3.13). The equation

$$
E_{4}=0,
$$

is the frequency equation of axially symmetric dilatational vibrations of the fluid-loaded poroelastic hollow cylinder for an impervious surface. Equation (4.7) shows that the shear and dilatational vibrations of the fluid-loaded poroelastic hollow cylinder surrounded by a fluid for an impervious surface are uncoupled.

From Eqs (4.5) and (4.9), it is clear that the frequency equation of axially symmetric shear vibrations of the fluid-loaded poroelastic hollow cylinder is same for a pervious and impervious surface. Hence, axially symmetric shear vibrations of the fluid-loaded poroelastic hollow cylinder are independent of the nature of the surface.

\subsubsection{Fluid-loaded poroelastic bore}

When the outer radius of the hollow poroelastic cylinder tends to infinity i.e., $r_{2} \rightarrow \infty$, then the fluidloaded poroelastic hollow cylinder reduces to a fluid-loaded poroelastic bore. In this case, the frequency Eq.(4.1) of the fluid-loaded poroelastic hollow cylinder for a pervious surface reduces to

$$
\left|\begin{array}{cccc}
C_{12} & C_{14} & C_{16} & C_{17} \\
C_{22} & C_{24} & C_{26} & 0 \\
C_{32} & C_{34} & C_{36} & C_{37} \\
C_{42} & C_{44} & 0 & C_{47}
\end{array}\right|=0,
$$

where the elements $C_{i j}$ are defined in Eq.(3.4).

This is the frequency equation of the fluid-loaded poroelastic bore for a pervious surface.

Similarly, the frequency Eq.(4.2) of the fluid-loaded poroelastic hollow cylinder for an impervious surface reduces to 


$$
\left|\begin{array}{cccc}
D_{12} & D_{14} & D_{16} & D_{17} \\
D_{22} & D_{24} & D_{26} & 0 \\
D_{32} & D_{34} & D_{36} & D_{37} \\
D_{42} & D_{44} & 0 & D_{47}
\end{array}\right|=0,
$$

where the elements $D_{i j}$ are defined in Eq.(3.6).

This is the frequency equation of the fluid-loaded poroelastic bore for an impervious surface.

\section{Axially symmetric vibrations $(n=0)$}

For axially symmetric vibrations, the frequency Eq.(4.11) of vibrations of the fluid-loaded poroelastic bore for a pervious surface reduces to

$$
G_{1} G_{2}=0 \text {, }
$$

with

$$
G_{2}=\left|\begin{array}{lll}
C_{12} & C_{14} & C_{17} \\
C_{32} & C_{34} & C_{37} \\
C_{42} & C_{44} & C_{47}
\end{array}\right|,
$$

where the elements $C_{i j}$ are defined in Eq.(3.4).

From Eq.(4.13) we obtain $G_{1}=0$ or $G_{2}=0$. On simplification, the equation

$$
G_{1}=0,
$$

reduces to $\quad Y_{0}\left(\xi_{3} r_{1}\right)=0$,

which is independent of fluid parameters and dilatational vibrations, hence this the frequency equation of axially symmetric shear vibrations of the poroelastic bore for a pervious surface. The equation

$$
G_{2}=0,
$$

is the frequency equation of axially symmetric dilatational vibrations of the fluid-loaded poroelastic bore for a pervious surface. Equation (4.13) shows that the axially symmetric shear and dilatational vibrations of the fluid-loaded poroelastic bore for a pervious surface are uncoupled.

Similarly, the frequency Eq.(4.12) of vibrations of the fluid-loaded poroelastic bore for an impervious surface reduces to

$$
G_{3} G_{4}=0,
$$

with

$$
G_{4}=\left|\begin{array}{lll}
D_{12} & D_{14} & D_{17} \\
D_{32} & D_{34} & D_{37} \\
D_{42} & D_{44} & D_{47}
\end{array}\right|
$$

where the elements $D_{i j}$ are defined in Eq.(3.6).

From Eq.(4.17) we obtain $G_{3}=0$ or $G_{4}=0$. On simplification, the equation

$$
G_{3}=0,
$$

reduces to $\quad Y_{0}\left(\xi_{3} r_{1}\right)=0$,

which is independent of fluid parameters and dilatational vibrations, hence this the frequency equation of axially symmetric shear vibrations of the poroelastic bore for an impervious surface. The equation

$$
G_{4}=0,
$$


is the frequency equation of axially symmetric dilatational vibrations of the fluid-loaded poroelastic bore for an impervious surface. Equation (4.17) shows that the axially symmetric shear and dilatational vibrations of the fluid-loaded poroelastic bore for a pervious surface are uncoupled.

From Eqs (4.15a) and (4.17), it is clear that the frequency equation of axially symmetric shear vibrations of the fluid-loaded poroelastic bore is same for a pervious and an impervious surface. Hence, axially symmetric shear vibrations of the fluid-loaded poroelastic bore are independent of the nature of the surface.

\subsection{1a. Poroelastic bore}

When the fluid density $\rho_{f}^{(I)}$ of the inner fluid is zero, then the fluid-loaded poroelastic bore reduces to a poroelastic bore of radius $r_{l}$. In this case, the frequency Eq.(4.11) of the fluid-loaded poroelastic bore for a pervious surface reduces to

$$
\left|\begin{array}{ccc}
C_{12} & C_{14} & C_{16} \\
C_{22} & C_{24} & C_{26} \\
C_{42} & C_{44} & 0
\end{array}\right|=0
$$

where the elements $C_{i j}$ are defined in Eq.(3.4).

This is the frequency equation of the poroelastic bore for a pervious surface.

Similarly, the frequency Eq.(4.12) of the fluid-loaded poroelastic bore for an impervious surface reduces to

$$
\left|\begin{array}{ccc}
D_{12} & D_{14} & D_{16} \\
D_{22} & D_{24} & D_{26} \\
D_{42} & D_{44} & 0
\end{array}\right|=0
$$

where the elements $D_{i j}$ are defined in Eq.(3.6).

This is the frequency equation of the poroelastic bore for an impervious surface.

\section{Axially symmetric vibrations $(n=0)$}

For axially symmetric vibrations, the frequency Eq.(4.21) of vibrations of the poroelastic bore for a pervious surface reduces to

$$
I_{1} I_{2}=0
$$

with

$$
I_{1}=C_{26}, \quad I_{2}=\left|\begin{array}{ll}
C_{12} & C_{14} \\
C_{42} & C_{42}
\end{array}\right|
$$

where the elements $C_{i j}$ are defined in $\mathrm{Eq}(3.4)$.

From Eq.(4.23) we obtain $I_{1}=0$ or $I_{2}=0$. In particular, the equation

$$
I_{1}=0
$$

is the frequency equation of axially symmetric shear vibrations of the poroelastic bore for a pervious surface and is same as the equation $G_{l}=0$ obtained in Eq.(4.15). The equation

$$
I_{2}=0
$$

is the frequency equation of axially symmetric dilatational vibrations of the poroelastic bore for a pervious surface. Equation (4.23) shows that axially symmetric shear and dilatational vibrations of the poroelastic bore for a pervious surface are uncoupled.

Similarly, the frequency Eq.(4.22) of vibrations of the poroelastic bore for an impervious surface reduces to 


$$
I_{3} I_{4}=0
$$

with

$$
I_{3}=D_{26}, \quad I_{4}=\left|\begin{array}{ll}
D_{12} & D_{14} \\
D_{42} & D_{42}
\end{array}\right|
$$

where the elements $D_{i j}$ are defined in Eq.(3.6).

From Eq.(4.27) we obtain $I_{3}=0$ or $I_{4}=0$. In particular, the equation

$$
I_{3}=0
$$

is the frequency equation of axially symmetric shear vibrations of the poroelastic bore for an impervious surface and is same as the equation $G_{3}=0$ obtained in Eq.(4.19). The equation

$$
I_{4}=0,
$$

is the frequency equation of axially symmetric dilatational vibrations of the poroelastic bore for an impervious surface. Equation (4.27) shows that axially symmetric shear and dilatational vibrations of the poroelastic bore for a pervious surface are uncoupled. From Eqs (4.25) and (4.29), it is clear that the frequency equation of axially symmetric shear vibrations of the poroelastic bore is same for a pervious and an impervious surface. Hence, axially symmetric shear vibrations of the poroelastic bore are independent of the the nature of surface.

\subsection{Poroelastic hollow cylinder surrounded by fluid}

When the fluid density $\rho_{f}^{(I)}$ of the inner fluid is zero, then the fluid-loaded poroelastic hollow cylinder surrounded by a fluid reduces to a poroelastic hollow cylinder surrounded by a fluid. In this case, the frequency Eq.(3.3) of the fluid-loaded poroelastic hollow cylinder surrounded by a fluid for a pervious surface reduces to

$$
\left|\begin{array}{lllllll}
C_{11} & C_{12} & C_{13} & C_{14} & C_{15} & C_{16} & 0 \\
C_{21} & C_{22} & C_{23} & C_{24} & C_{25} & C_{26} & 0 \\
C_{41} & C_{42} & C_{43} & C_{44} & 0 & 0 & 0 \\
C_{51} & C_{52} & C_{53} & C_{54} & C_{55} & C_{56} & C_{58} \\
C_{61} & C_{62} & C_{63} & C_{64} & C_{65} & C_{66} & 0 \\
C_{71} & C_{72} & C_{73} & C_{74} & C_{75} & C_{76} & C_{78} \\
C_{81} & C_{82} & C_{83} & C_{84} & 0 & 0 & C_{88}
\end{array}\right|=0
$$

where the elements $C_{i j}$ are defined in Eq.(3.4).

This is the frequency equation of the poroelastic hollow cylinder surrounded by a fluid for a pervious surface.

In a similar way, the frequency Eq.(3.5) of the fluid-loaded poroelastic hollow cylinder surrounded by fluid for an impervious surface reduces to

$$
\left|\begin{array}{lllllll}
D_{11} & D_{12} & D_{13} & D_{14} & D_{15} & D_{16} & 0 \\
D_{21} & D_{22} & D_{23} & D_{24} & D_{25} & D_{26} & 0 \\
D_{41} & D_{42} & D_{43} & D_{44} & 0 & 0 & 0 \\
D_{51} & D_{52} & D_{53} & D_{54} & D_{55} & D_{56} & D_{58} \\
D_{61} & D_{62} & D_{63} & D_{64} & D_{65} & D_{66} & 0 \\
D_{71} & D_{72} & D_{73} & D_{74} & D_{75} & D_{76} & D_{78} \\
D_{81} & D_{82} & D_{83} & D_{84} & 0 & 0 & D_{88}
\end{array}\right|=0
$$


where the elements $D_{i j}$ are defined in Eq.(3.6).

This is the frequency equation of the poroelastic hollow cylinder surrounded by a fluid for an impervious surface.

\section{Axially symmetric vibrations $(n=0)$}

For axially symmetric vibrations, the frequency Eq.(4.31) of vibrations of the poroelastic hollow cylinder surrounded by a fluid for a pervious surface reduces to

with

$$
M_{1} M_{2}=0, \quad \begin{array}{ll}
C_{25} & C_{26} \\
C_{65} & C_{66}
\end{array}\left|, \quad M_{2}=\right| \begin{array}{lllll}
C_{11} & C_{12} & C_{13} & C_{14} & 0 \\
C_{41} & C_{42} & C_{43} & C_{44} & 0 \\
C_{51} & C_{52} & C_{53} & C_{54} & C_{58} \\
C_{71} & C_{72} & C_{73} & C_{74} & C_{78} \\
C_{81} & C_{82} & C_{83} & C_{84} & C_{88}
\end{array} \mid
$$

where the elements $C_{i j}$ are defined in Eq.(3.4).

From Eq.(4.33) we obtain $M_{1}=0$ or $M_{2}=0$. In particular, the equation

$$
M_{1}=0,
$$

is the frequency equation of axially symmetric shear vibrations of the poroelastic hollow cylinder for a pervious surface and is same as the equation $A_{1}=0$ obtained in Eq.(3.9). The equation

$$
M_{2}=0 \text {, }
$$

is the frequency equation of axially symmetric dilatational vibrations of the poroelastic hollow cylinder surrounded by a fluid for a pervious surface. Equation (4.33) shows that the shear and dilatational vibrations of the fluid-loaded poroelastic hollow cylinder for a pervious surface are uncoupled.

Similarly, the frequency Eq.(4.32) of vibrations of the poroelastic hollow cylinder surrounded by a fluid for an impervious surface reduces to

$$
\begin{aligned}
& M_{3} M_{4}=0, \quad M_{3}=\left|\begin{array}{ll}
D_{25} & D_{26} \\
D_{65} & D_{66}
\end{array}\right|, \quad M_{4}=\left|\begin{array}{lllll}
D_{11} & D_{12} & D_{13} & D_{14} & 0 \\
D_{41} & D_{42} & D_{43} & D_{44} & 0 \\
D_{51} & D_{52} & D_{53} & D_{54} & D_{58} \\
D_{71} & D_{72} & D_{73} & D_{74} & D_{78} \\
D_{81} & D_{82} & D_{83} & D_{84} & D_{88}
\end{array}\right|
\end{aligned}
$$

with

where the elements $D_{i j}$ are defined in Eq.(3.6).

From Eq.(4.37), it is clear that $M_{3}=0$ or $M_{4}=0$. In particular, the equation

$$
M_{3}=0
$$

is the frequency equation of axially symmetric shear vibrations of the poroelastic hollow cylinder surrounded by a fluid for an impervious surface and is same as the equation $B_{1}=0$ obtained in Eq.(3.13). The equation

$$
M_{4}=0 \text {, }
$$

is the frequency equation of axially symmetric dilatational vibrations of the poroelastic hollow cylinder surrounded by a fluid for an impervious surface. Equation (4.37) shows that the shear and dilatational vibrations of the poroelastic hollow cylinder surrounded by a fluid for an impervious surface are uncoupled. 
From Eqs (4.35) and (4.39), it is clear that the frequency equation of axially symmetric shear vibrations of the poroelastic hollow cylinder surrounded by a fluid is same for a pervious and an impervious surface. Hence, axially symmetric shear vibrations of the poroelastic hollow cylinder surrounded by a fluid are independent of the nature of the surface.

\subsubsection{Poroelastic solid cylinder submerged in a fluid}

When the inner radius of the hollow poroelastic cylinder tends to zero i.e., $r_{l} \rightarrow 0$ then the poroelastic hollow cylinder surrounded by a fluid reduces to a poroelastic solid cylinder submerged in fluid. In this case, the frequency Eq.(4.31) of the poroelastic hollow cylinder surrounded by a fluid for a pervious surface reduces to

$$
\left|\begin{array}{llll}
C_{51} & C_{53} & C_{55} & C_{58} \\
C_{61} & C_{63} & C_{65} & 0 \\
C_{71} & C_{73} & C_{75} & C_{78} \\
C_{81} & C_{83} & 0 & C_{88}
\end{array}\right|=0
$$

where the elements $C_{i j}$ are defined in Eq.(3.4).

This is the frequency equation of vibrations of the poroelastic solid cylinder submerged in a fluid for a pervious surface.

Similarly, the frequency Eq.(4.32) of the poroelastic hollow cylinder surrounded by a fluid for an impervious surface reduces to

$$
\left|\begin{array}{llll}
D_{51} & D_{53} & D_{55} & D_{58} \\
D_{61} & D_{63} & D_{65} & 0 \\
D_{71} & D_{73} & D_{75} & D_{78} \\
D_{81} & D_{83} & 0 & D_{88}
\end{array}\right|=0
$$

where the elements $D_{i j}$ are defined in Eq.(3.6).

This is the frequency equation of vibrations of the poroelastic solid cylinder submerged in a fluid for an impervious surface.

\section{Axially symmetric vibrations $(n=0)$}

For axially symmetric vibrations, the frequency Eq.(4.41) of vibrations of the poroelastic solid cylinder submerged in a fluid for a pervious surface reduces to

$$
P_{1} P_{2}=0,
$$

with $\quad P_{1}=C_{65}, \quad P_{2}=\left|\begin{array}{lll}C_{51} & C_{53} & C_{58} \\ C_{71} & C_{73} & C_{78} \\ C_{81} & C_{83} & C_{88}\end{array}\right|$

where the elements $C_{i j}$ are defined in Eq.(3.4).

From Eq.(4.43) we obtain $P_{1}=0$ or $P_{2}=0$. On simplification, the equation

$$
P_{1}=0,
$$

reduces to $\quad J_{0}\left(\xi_{3} r_{2}\right)=0$, 
which is independent of fluid parameters and dilatational vibrations, hence this is the frequency equation of axially symmetric shear vibrations of the poroelastic solid cylinder for a pervious surface. The equation

$$
P_{2}=0,
$$

is the frequency equation of axially symmetric dilatational vibrations of the poroelastic solid cylinder submerged in a fluid for a pervious surface. Equation (4.43) shows that the axially symmetric shear and dilatational vibrations of the poroelastic solid cylinder submerged in a fluid for a pervious surface are uncoupled.

Similarly, the frequency Eq.(4.42) of vibrations of the poroelastic solid cylinder submerged in a fluid for an impervious surface reduces to

$$
P_{3} P_{4}=0,
$$

with $\quad P_{3}=D_{65}, \quad P_{4}=\left|\begin{array}{ccc}D_{51} & D_{53} & D_{58} \\ D_{71} & D_{73} & D_{78} \\ D_{81} & D_{83} & D_{88}\end{array}\right|$

where the elements $D_{i j}$ are defined in Eq.(3.6).

From Eq.(4.47) we obtain $P_{3}=0$ or $P_{4}=0$. On simplification, the equation

$$
P_{3}=0,
$$

reduces to

$$
J_{0}\left(\xi_{3} r_{2}\right)=0
$$

which is independent of fluid parameters and dilatational vibrations, hence this the frequency equation of axially symmetric shear vibrations of the poroelastic solid cylinder for an impervious surface. The equation

$$
P_{4}=0,
$$

is the frequency equation of axially symmetric dilatational vibrations of the poroelastic solid cylinder submerged in a fluid for an impervious surface. Equation (4.47) shows that the axially symmetric shear and dilatational vibrations of the poroelastic solid cylinder submerged in a fluid for a pervious surface are uncoupled.

From Eqs (4.45a) and (4.49a), it is clear that the frequency equation of axially symmetric shear vibrations of the poroelastic solid cylinder submerged in a fluid is same for a pervious and an impervious surface. Hence, axially symmetric shear vibrations of the poroelastic solid cylinder submerged in a fluid are independent of the nature of the surface.

\section{Nondimensionalization of the frequency equation}

To analyze the frequency equations obtained above, it is convenient to introduce the following nondimensional parameters

$$
\begin{aligned}
& a_{1}=\frac{P}{H}, \quad a_{2}=\frac{Q}{H}, \quad a_{3}=\frac{R}{H}, \quad a_{4}=\frac{N}{H}, \quad d_{1}=\frac{\rho_{11}}{\rho}, \quad d_{2}=\frac{\rho_{12}}{\rho}, \quad d_{3}=\frac{\rho_{22}}{\rho}, \\
& x_{1}=\left(\frac{V_{0}}{V_{1}}\right)^{2}, \quad y_{1}=\left(\frac{V_{0}}{V_{2}}\right)^{2}, \quad z_{1}=\left(\frac{V_{0}}{V_{3}}\right)^{2}, \quad \Omega=\frac{\omega h}{C_{0}}, \quad C=\frac{\omega}{k}, \quad m=\frac{C}{C_{0}}, \\
& m_{1}=\frac{\rho_{f}^{(l)}}{\rho}, \quad m_{2}=\frac{\rho_{f}^{(2)}}{\rho}, \quad t_{1}=\frac{V_{f}^{(l)}}{V_{3}}, \quad \text { and } \quad t_{2}=\frac{V_{f}^{(2)}}{V_{3}} .
\end{aligned}
$$


In Eq.(5.1), $\Omega$ is the non-dimensional frequency, $C$ is phase velocity, $m$ is non-dimensional phase velocity, $C_{0}$ and $V_{0}$ are reference velocities $\left(C_{0}^{2}=N \rho^{-1}, V_{0}^{2}=H \rho^{-1}\right), H=P+2 Q+R$ and

$$
\rho=\rho_{11}+2 \rho_{12}+\rho_{22}
$$

\section{Results and discussion}

Two types of poroelastic cylinders are considered to carry out the computational work, one is sandstone saturated with water, say, cylinder I (Yew and Jogi, 1976), the other one is sandstone saturated with kerosene, say, cylinder II (Fatt, 1959), whose non-dimensional physical parameters are given in Tab.1.

Table.1.

\begin{tabular}{|c|c|c|c|c|c|c|c|c|c|c|}
\hline $\begin{array}{c}\text { Material } \\
\text { Parameters }\end{array}$ & $a_{1}$ & $a_{2}$ & $a_{3}$ & $a_{4}$ & $d_{1}$ & $d_{2}$ & $d_{3}$ & $x_{1}$ & $y_{1}$ & $z_{1}$ \\
\hline Cylinder I & 0.960 & 0.006 & 0.028 & 0.412 & 0.877 & 0 & 0.123 & 0.913 & 4.347 & 2.129 \\
\hline Cylinder II & 0.843 & 0.065 & 0.028 & 0.234 & 0.901 & -0.001 & 0.101 & 0.999 & 4.763 & 3.851 \\
\hline
\end{tabular}

For given poroelastic materials, the above obtained frequency equations, when non-dimensionalized using Eq.(5.1), constitute a relation between the frequency and the ratio of thickness to the inner radius or a relation between phase velocity and wavenumber. Non-dimensional frequency is determined each for a pervious and an impervious surface in the case of axially symmetric, flexural and anti-symmetric vibrations. The non-dimensional frequency is computed for different values of the ratio of wall thickness to the inner radius $h / r_{l}$. The values of $h / r_{l}$ lie in $\left[\begin{array}{ll}0.1 & 1.2\end{array}\right]$ that represent a thin and moderately thick poroelastic cylinder. For the fluid-loaded poroelastic cylinder surrounded by a fluid, the internal fluid parameters are taken as $m_{1}=0.4, t_{1}=2.5$, while the external fluid parameters are taken as $m_{2}=0.9$, and $t_{2}=1.5$. In all other particular cases where only one fluid is involved, the fluid parameters $m_{1}$ or $m_{2}=0.4$ and $t_{1}$ or $t_{2}=2.5$ are taken.

The frequency of the fluid-loaded poroelastic hollow cylinder surrounded by a fluid for axially symmetric dilatational, flexural and anti-symmetric vibrations is presented in Figs 2, 3 and 4, respectively, for both cylinders I and II each for a pervious and an impervious surface. From Fig. 2 it is clear that the variation of frequency for cylinder I is more than that of cylinder II for a pervious surface and the frequency of cylinder II is higher than that of cylinder I for an impervious surface beyond the point $h / r_{l}=0.7$. In addition, the frequency of cylinder II is steady beyond $h / r_{1}=0.6$ for a pervious surface. The frequency of both cylinders I and II each for a pervious and an impervious surface is steady for $h / r_{1}>0.6$ in the case of flexural vibrations as shown in Fig.3. In addition, the frequency for an impervious surface is slightly higher than that of a pervious surface for cylinder I, while in the case of cylinder II, the frequency for an impervious surface is slightly less than that of a pervious surface beyond $h / r_{l}=0.6$. Frequency of the fluid-loaded poroelastic cylinder surrounded by a fluid for anti-symmetric vibrations is presented in Fig.4. It is observed that the frequency of both the cylinders I and II each for a pervious and an impervious surface is almost steady for $h / r_{1}>1$.

The variation of frequency for the fluid-loaded poroelastic hollow cylinder is shown in Figs 5-7 for axially symmetric dilatational, flexural and anti-symmetric vibrations, respectively. From Fig. 5 it is clear that the frequency of the fluid-loaded poroelastic cylinder I for an impervious surface is slightly higher than that of a pervious surface in $0.2<h / r_{l}<0.7$ and much higher beyond $h / r_{l}=0.7$. In addition, the frequency for both pervious and impervious surfaces is steady beyond $h / r_{l}=0.2$. The frequency of the fluid-loaded poroelastic cylinder II for a pervious and an impervious surface is nearly same and increasing steadily in the case of axially symmetric dilatational vibrations. Figure 6 shows that the frequency of the fluid-loaded poroelastic cylinder I for an impervious surface is higher than that of a pervious surface when $h / r_{l}>0.6$, while a reverse 
phenomenon is observed for poroelastic cylinder II in the case of flexural vibrations. Also, the frequency for both the cylinders I and II is steady and increasing beyond $h / r_{l}>0.7$. From Fig.7, it is observed that the variation in frequency for the fluid-loaded poroelastic cylinder II is more than that of cylinder I and the frequency for a pervious surface is same as that of an impervious surface beyond the point $h / r_{l}=0.5$ for the fluid-loaded poroelastic cylinder I in the case of anti-symmetric vibrations.

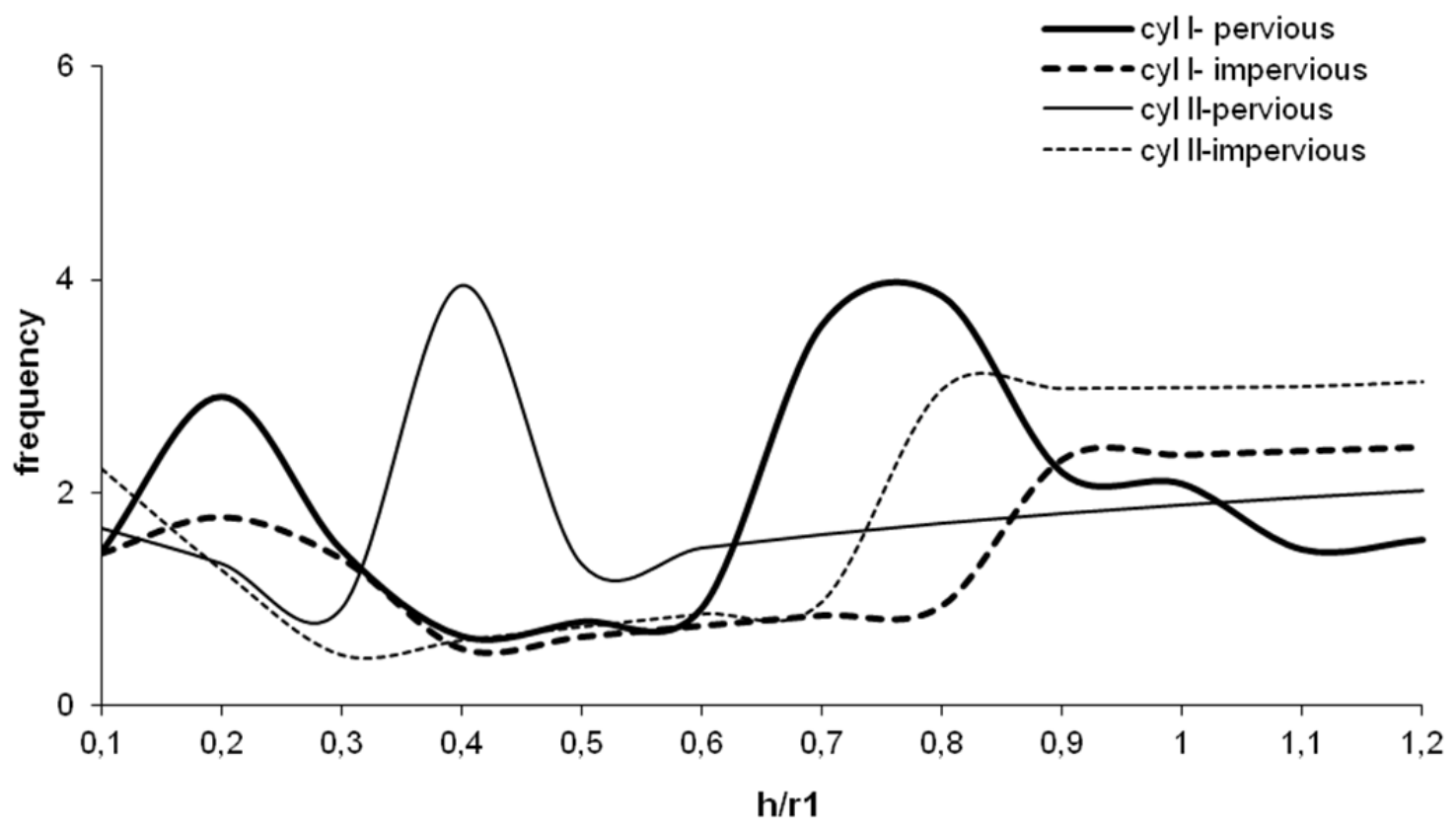

Fig.2. Frequency as a function of ratio of thickness to inner radius - fluid-loaded poroelastic hollow cylinder surrounded by a fluid - axially symmetric dilatational vibrations $(n=0)$.

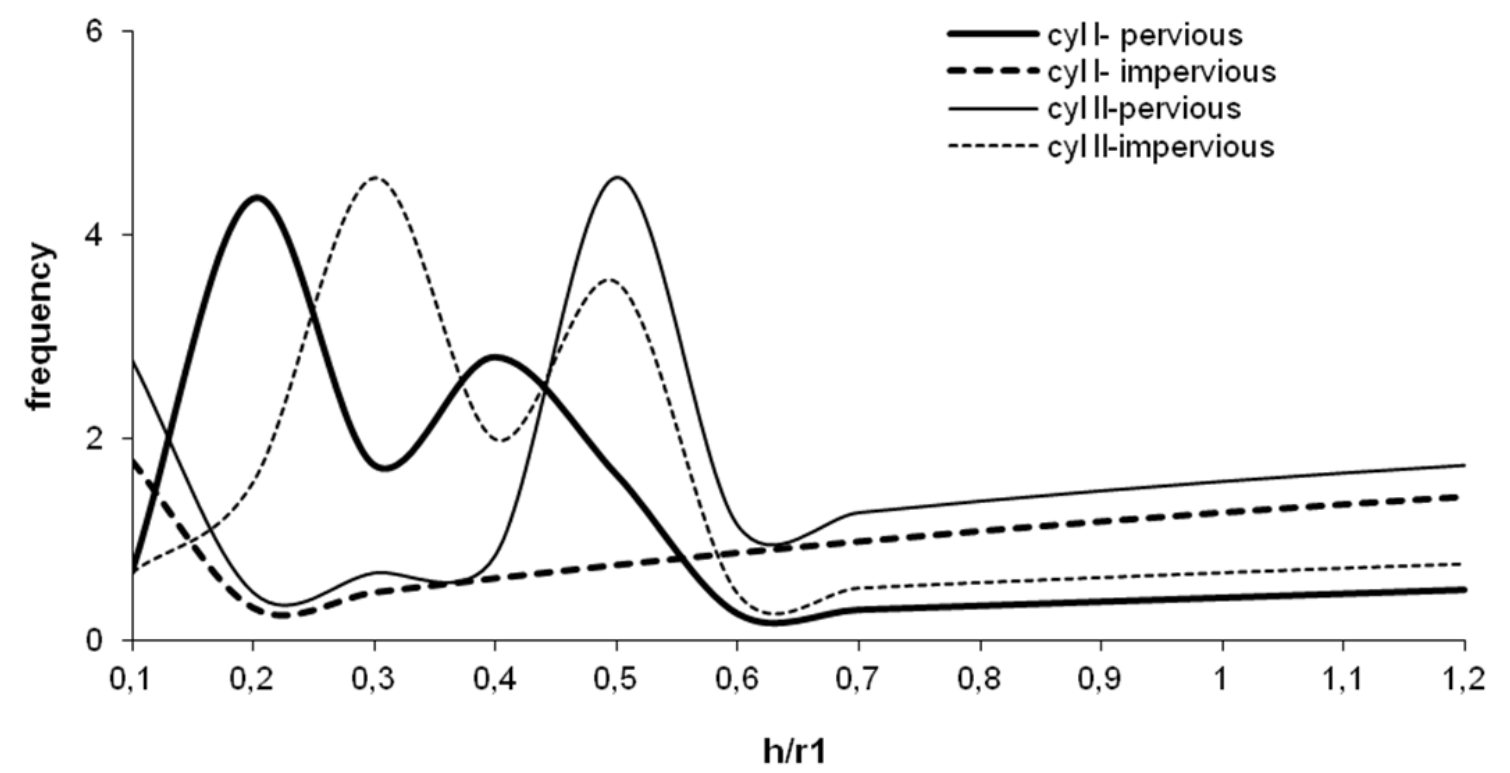

Fig.3. Frequency as a function of ratio of thickness to inner radius - fluid-loaded poroelastic hollow cylinder surrounded by a fluid - flexural vibrations $(n=1)$. 


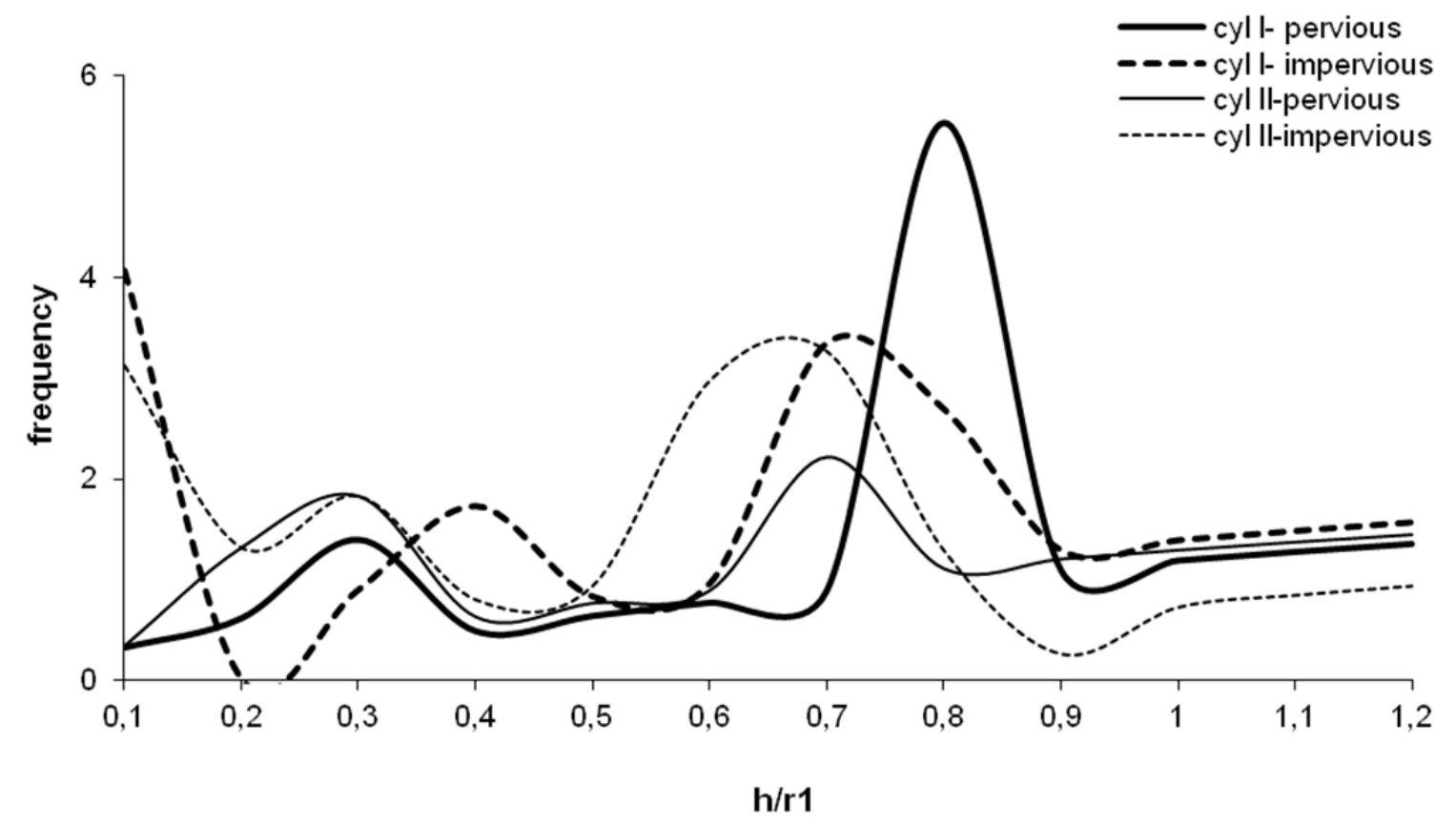

Fig.4. Frequency as a function of ratio of thickness to inner radius - fluid-loaded poroelastic hollow cylinder surrounded by a fluid - anti symmetric vibrations $(n=2)$.

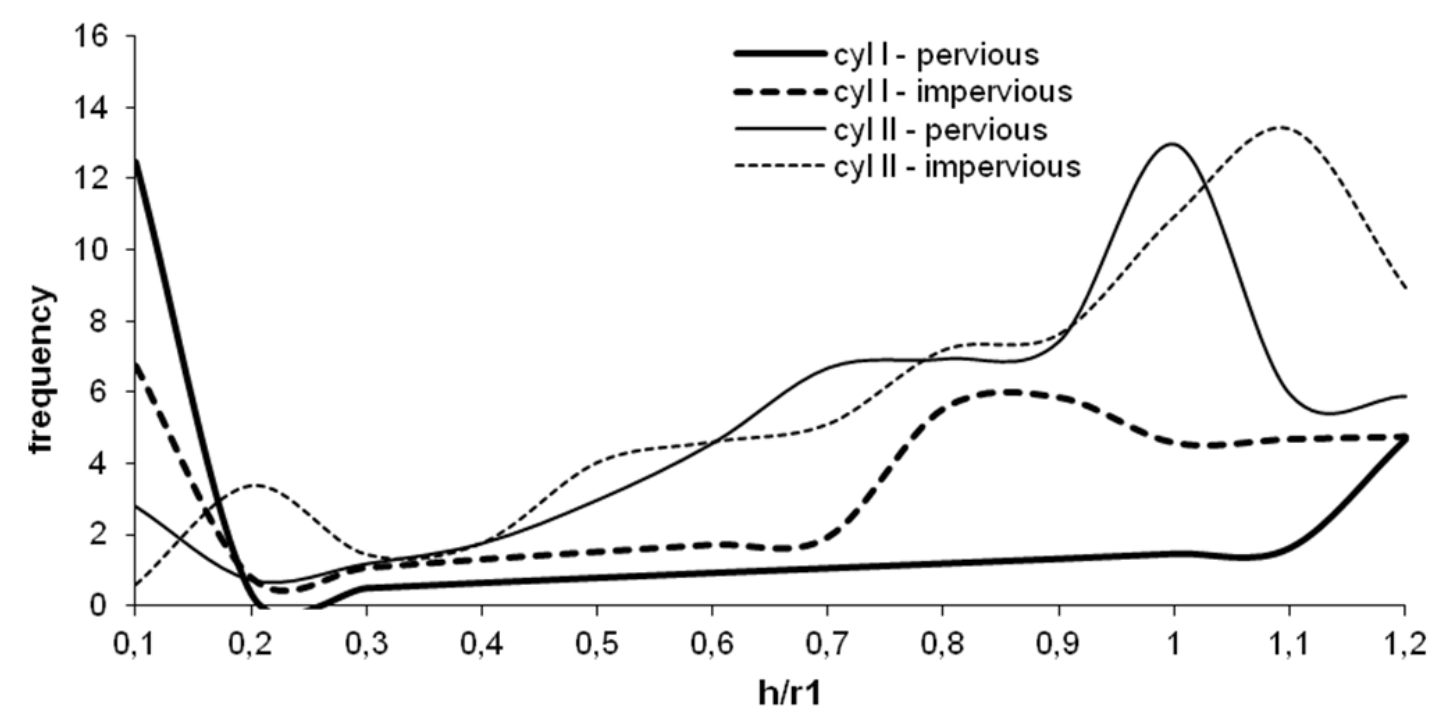

Fig.5. Frequency as a function of ratio of thickness to inner radius - fluid-loaded poroelastic hollow cylinder - axially symmetric dilatational vibrations $(n=0)$. 


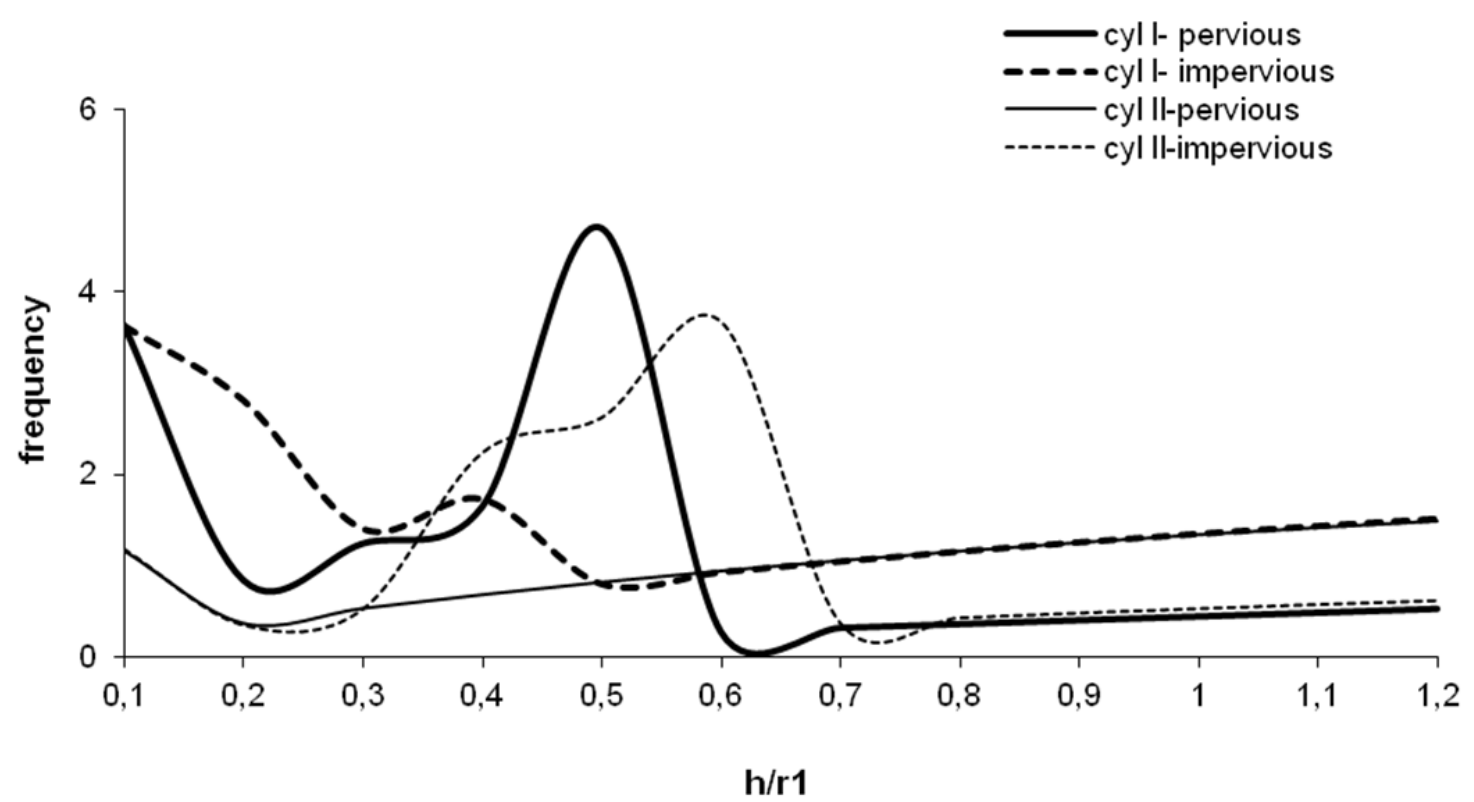

Fig.6. Frequency as a function of ratio of thickness to inner radius - fluid-loaded poroelastic hollow cylinder - flexural vibrations $(n=1)$.

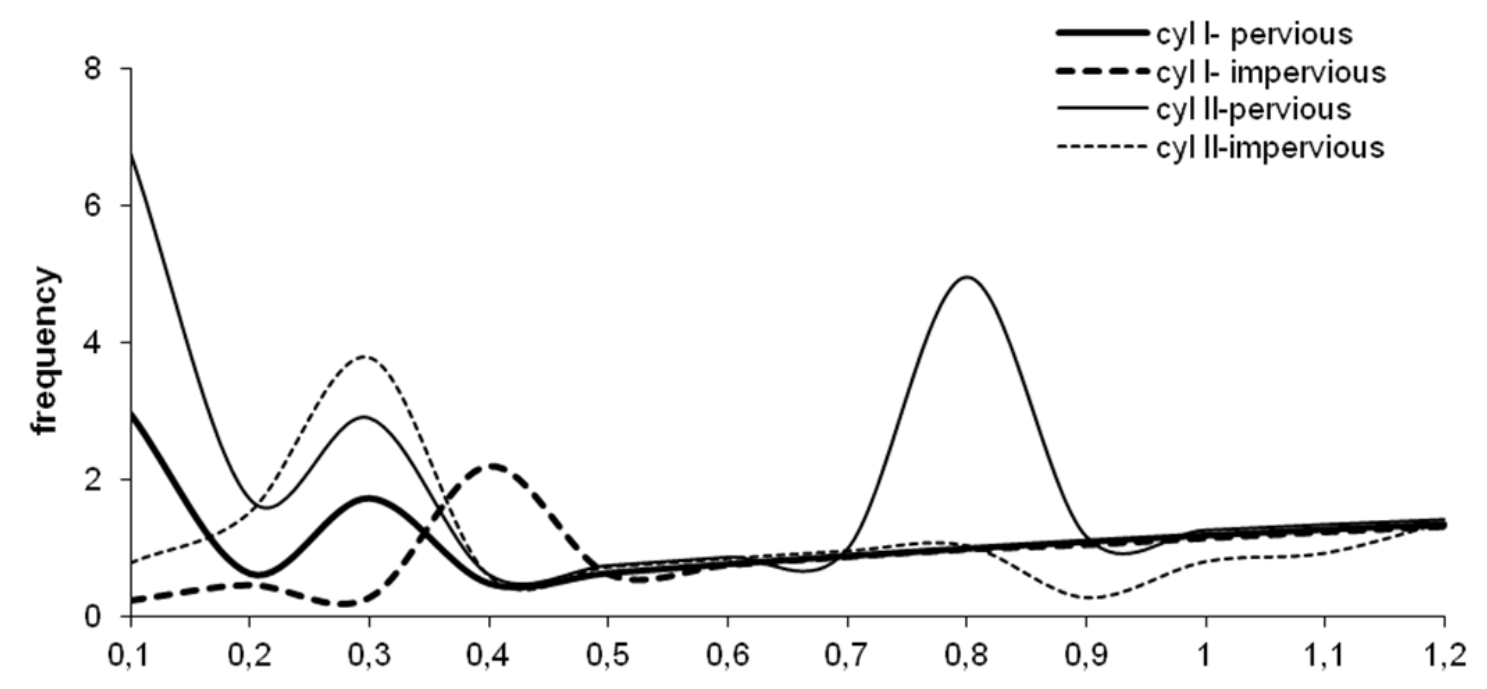

$\mathrm{h} / \mathrm{r} \mathbf{1}$

Fig.7. Frequency as a function of ratio of thickness to inner radius - fluid-loaded poroelastic hollow cylinder - anti symmetric vibrations $(n=2)$.

The phase velocity of the fluid-loaded poroelastic bore for axially symmetric dilatational vibrations is presented in Fig.8, for both cylinders I and II each for a pervious and an impervious surface. Figure 8 shows that the phase velocity of fluid-loaded poroelastic bore I is steadily decreasing for a pervious surface in case of axially symmetric dilatational vibrations. The phase velocity for a pervious surface is higher than that of impervious surface for the fluid-loaded poroelastic bore II. In addition, the variation of phase velocity for the the fluid-loaded poroelastic bore II is more when compared to the fluid-loaded poroelastic bore I. 


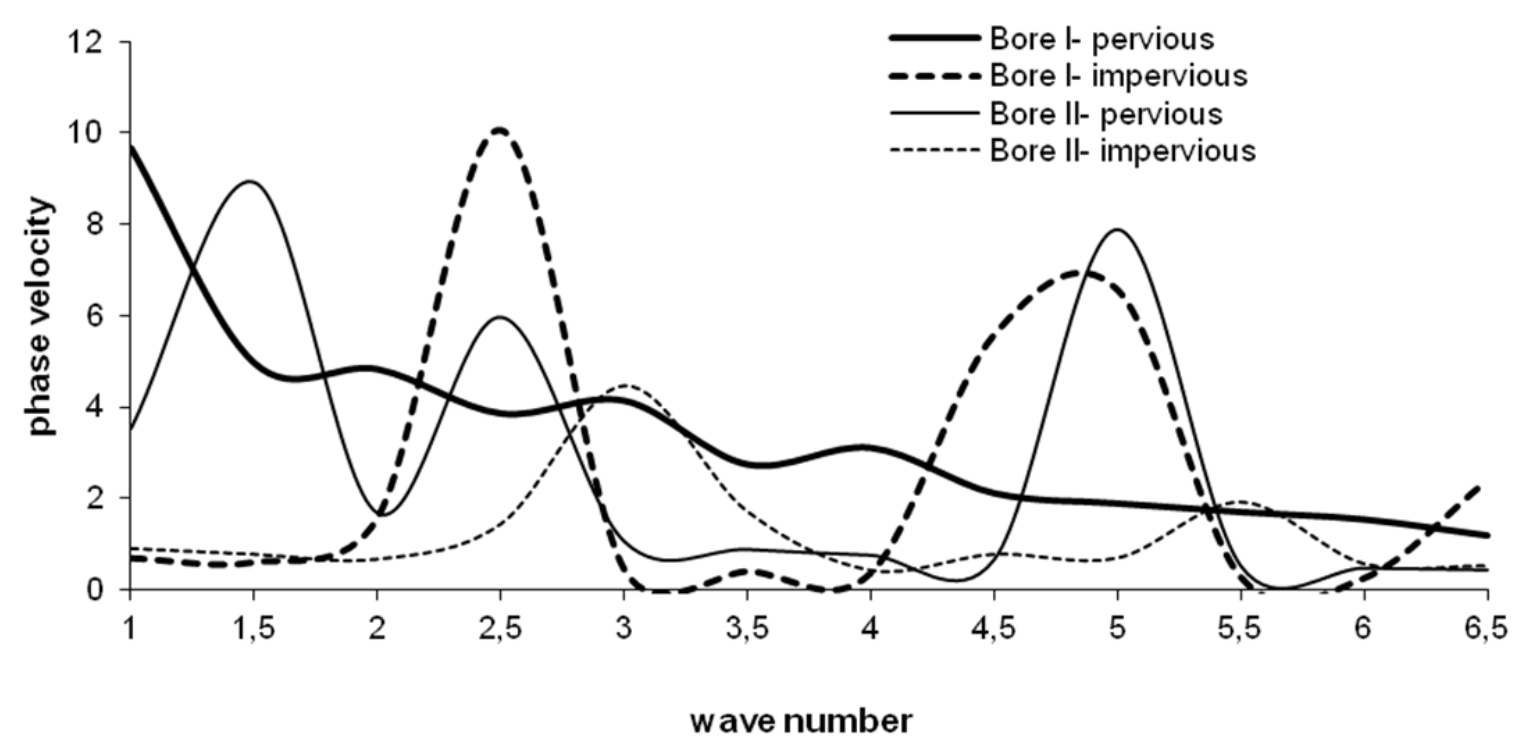

Fig.8. Phase velocity as a function of wavenumber - fluid-loaded poroelastic bore - axially symmetric dilatational vibrations $(n=0)$.

The variation of phase velocity for the poroelastic bore is depicted in Fig.9 for axially symmetric dilatational vibrations. From Fig.9, it is observed that phase velocity of the poroelastic bore I is more when compared to the poroelastic bore II in the case of axially symmetric dilatational vibrations. Figures 10-12 depict the frequency of the poroelastic hollow cylinder surrounded by a fluid in the case of axially symmetric, flexural and anti-symmetric vibrations, respectively. From Fig.10, it is observed that the frequency of the poroelastic hollow cylinder I surrounded by a fluid for an impervious surface is higher than that of pervious surface in $0.1<h / r_{1}<0.5$ and is lower beyond $h / r_{l}=0.5$ in the case of axially symmetric vibrations. For the poroelastic hollow cylinder II surrounded by a fluid, the frequency for a pervious surface is slightly higher than that of an impervious surface for $h / r_{l}>0.3$. The frequency of poroelastic cylinders I and II surrounded by a fluid is steady each for a pervious and an impervious surface beyond $h / r_{l}=0.5$. Figure 11 shows that the variation of frequency of the poroelastic hollow cylinder I surrounded by a fluid for a pervious surface is more when compared to an impervious surface, in addition the frequency is steady for the latter. The frequency of the poroelastic hollow cylinder II for a pervious surface is steady and less when compared to the frequency for an impervious surface in $0.15<h / r_{1}<0.57$ in the case of flexural vibrations. The variation of frequency for an impervious surface is more when compared to the frequency of a pervious surface for both poroelastic hollow cylinders I and II surrounded by a fluid in the case of anti-symmetric vibrations as shown in Fig.12. In addition, the frequency of the poroelastic hollow cylinder I and II surrounded by a fluid for a pervious surface is steady and almost same beyond $h / r_{l}=0.3$.

Figures 13-15 depict the frequency of the poroelastic hollow cylinder in the case of axially symmetric dilatational, flexural and anti-symmetric vibrations, respectively. From Fig. 13 it is clear that the frequency of both poroelastic hollow cylinders I and II is steady and increasing and the frequency of the poroelastic hollow cylinder I is same for a pervious and an impervious surface, whereas for cylinder II, the frequency for an impervious surface is slightly higher than that for a pervious surface. Thus, the variation of frequency of the poroelastic hollow cylinder I is independent of the surface. In addition, the frequency for the poroelastic hollow cylinder II is higher than that of cylinder I. Figure 14 shows that the frequencies of the poroelastic hollow cylinder I for an impervious surface and poroelastic hollow cylinder II for a pervious surface are same, steady and increasing in the case of flexural vibrations. The variation of frequency for a pervious surface is more compared to an impervious surface for both poroelastic hollow cylinders I and II in the case of anti-symmetric vibrations as shown in Fig.15. Further, the frequency of the poroelastic hollow cylinder I is same for a pervious and impervious surface beyond $h / r_{l}=0.5$. 


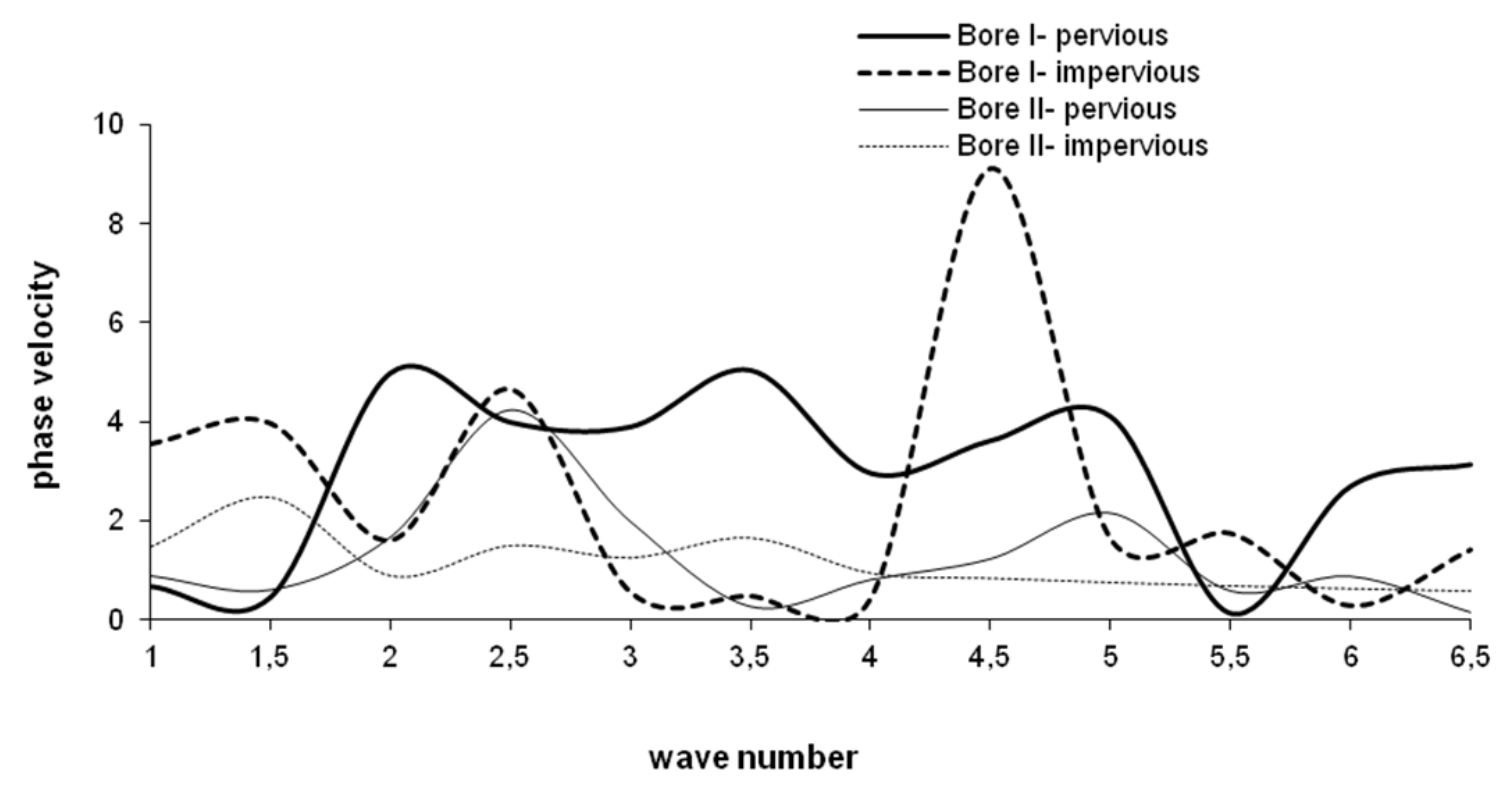

Fig.9. Phase velocity as a function of wavenumber - poroelastic bore - axially symmetric dilataional vibrations $(n=0)$.

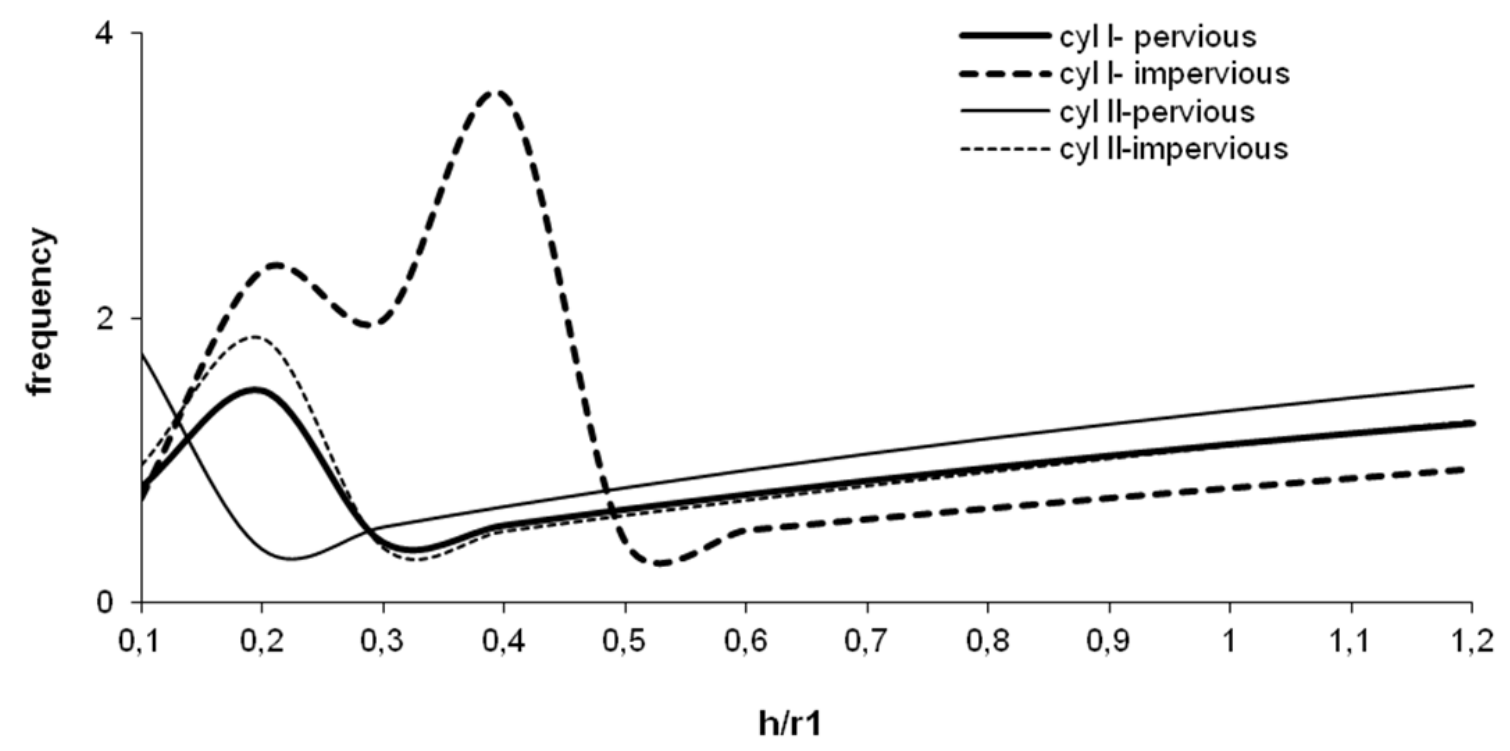

Fig.10. Frequency as a function of ratio of thickness to inner radius - poroelastic hollow cylinder surrounded by a fluid - axially symmetric dilatational vibrations $(n=0)$. 


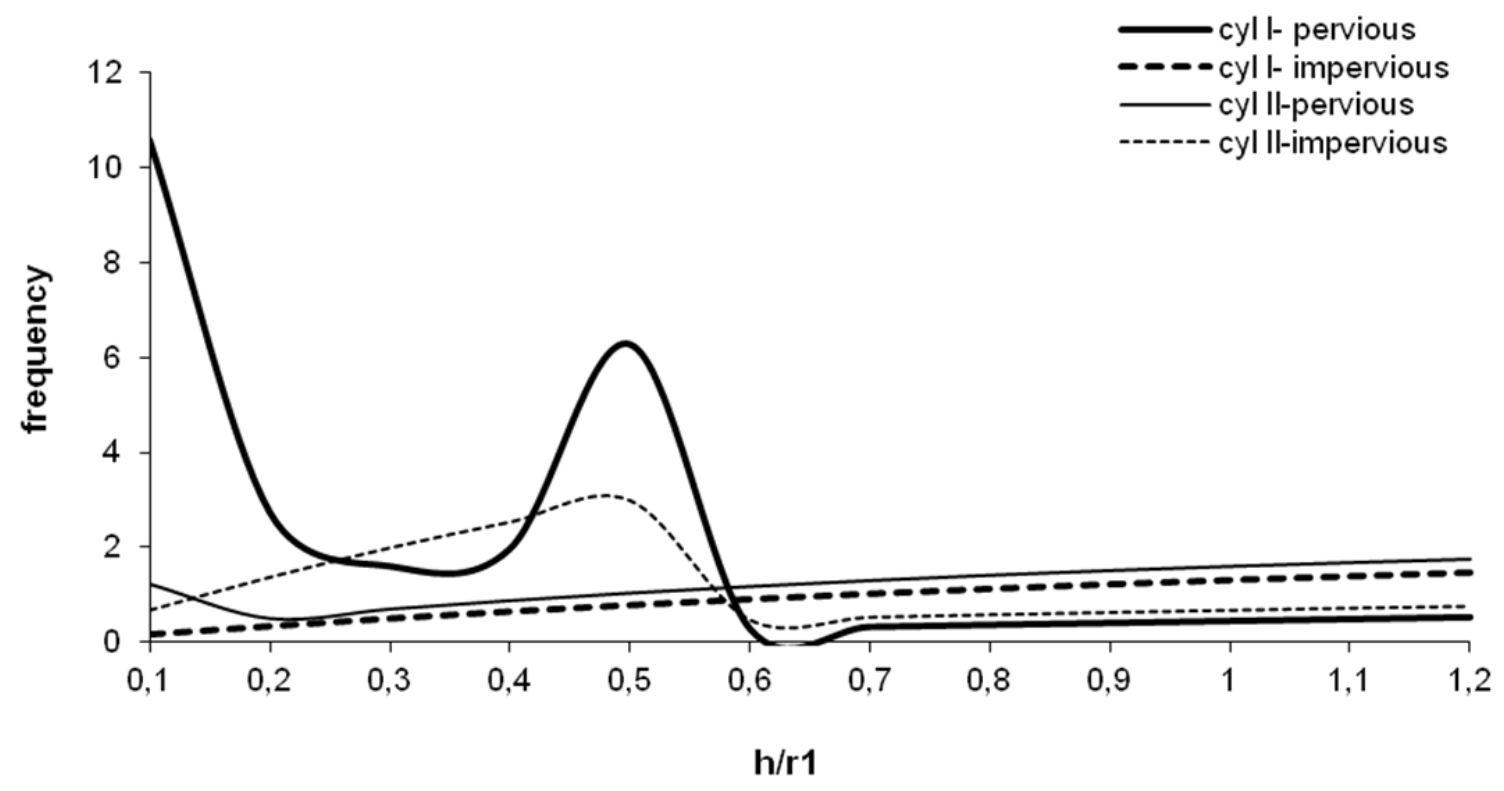

Fig.11. Frequency as a function of ratio of thickness to inner radius - poroelastic hollow cylinder surrounded by a fluid - flexural vibrations $(n=1)$.

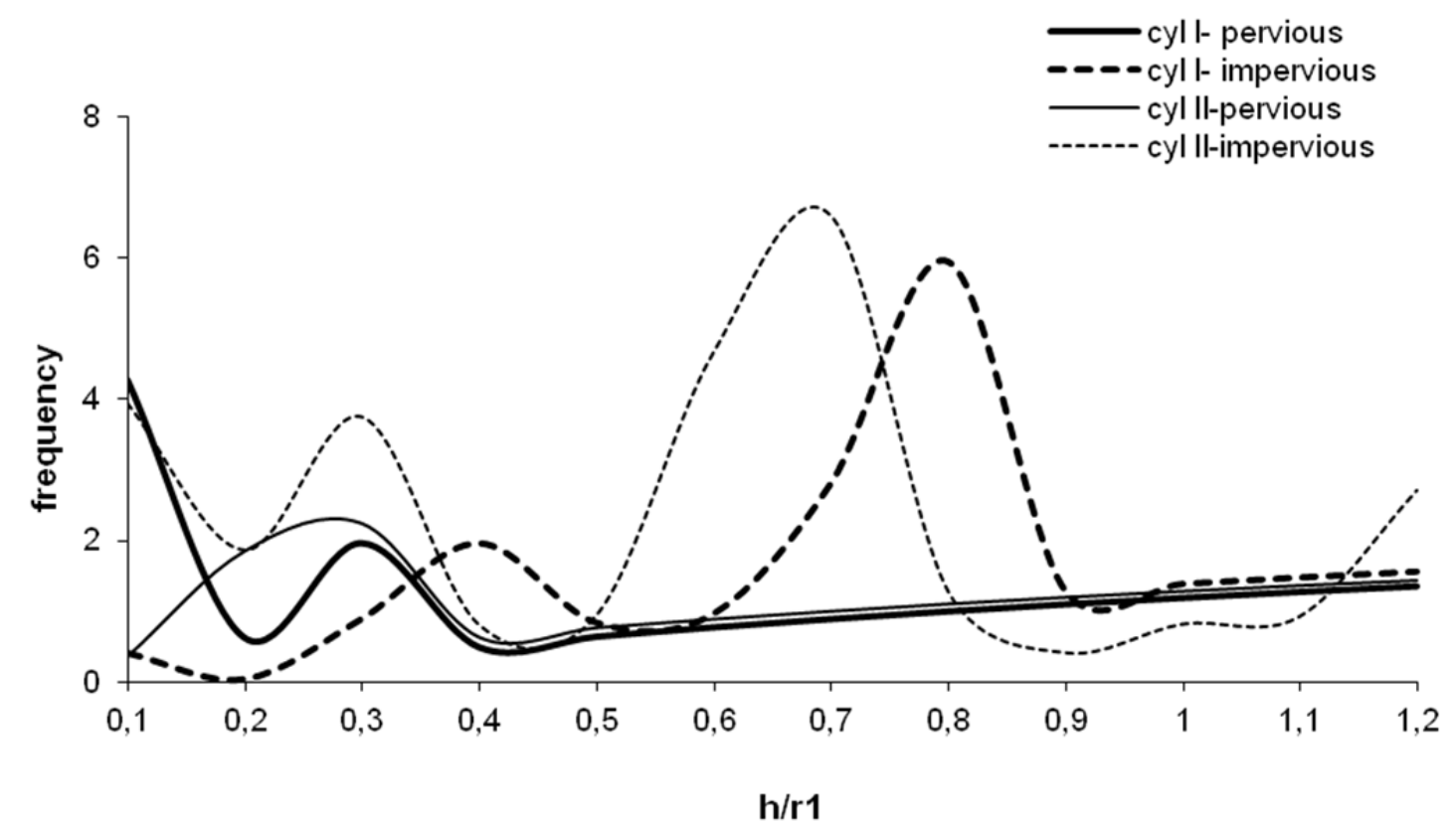

Fig.12. Frequency as a function of ratio of thickness to inner radius - poroelastic hollow cylinder surrounded by a fluid - anti symmetric vibrations $(n=2)$. 


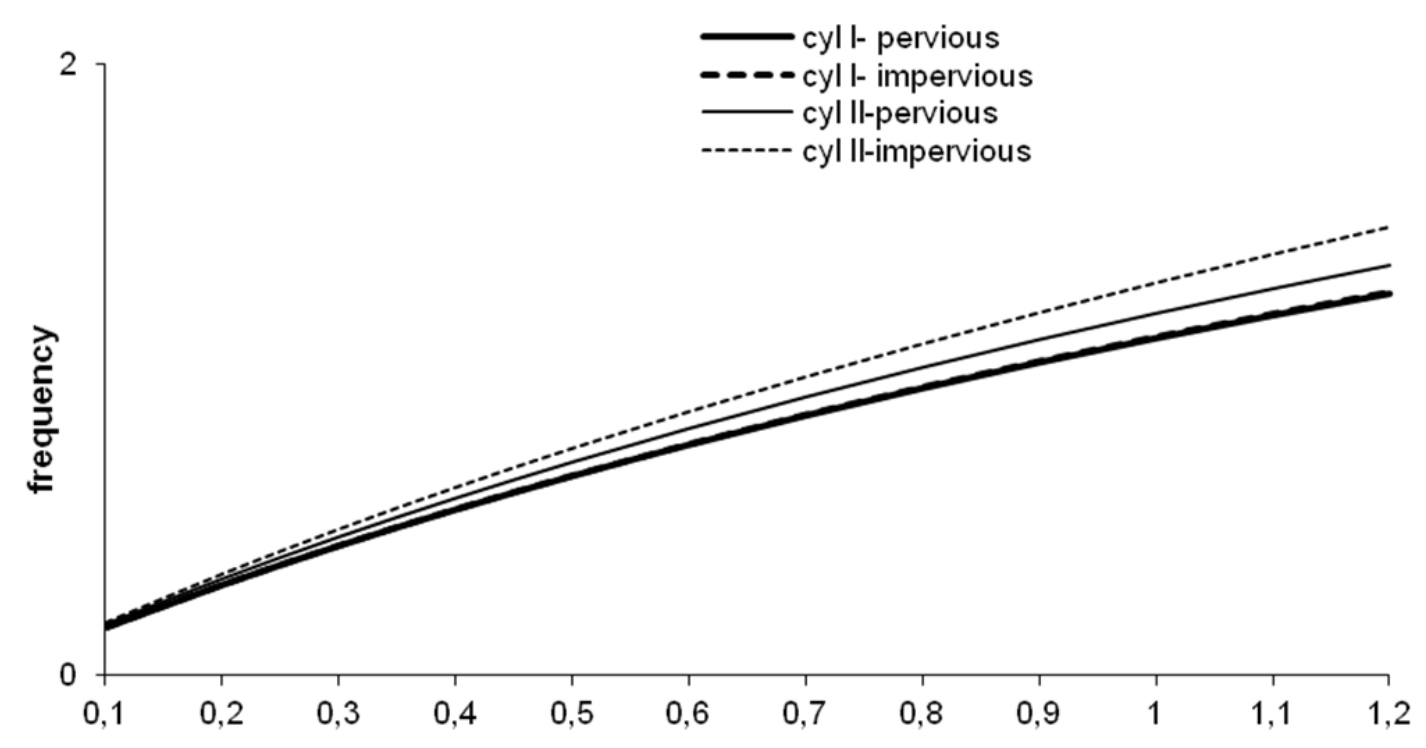

$\mathrm{h} / \mathrm{r} \mathbf{1}$

Fig.13. Frequency as a function of ratio of thickness to inner radius - poroelastic hollow cylinder - axially symmetric dilatational vibrations $(n=0)$.

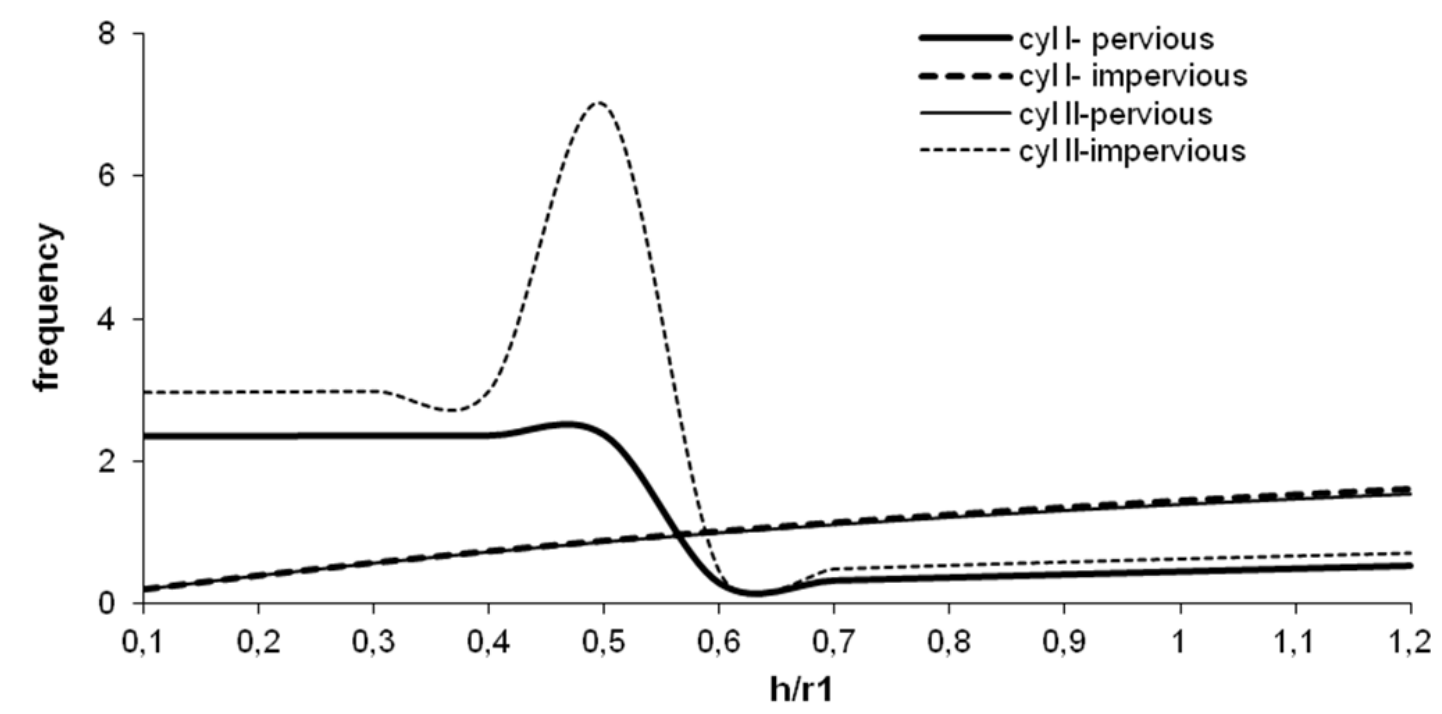

Fig.14. Frequency as a function of ratio of thickness to inner radius - poroelastic hollow cylinder - flexural vibrations $(n=1)$. 


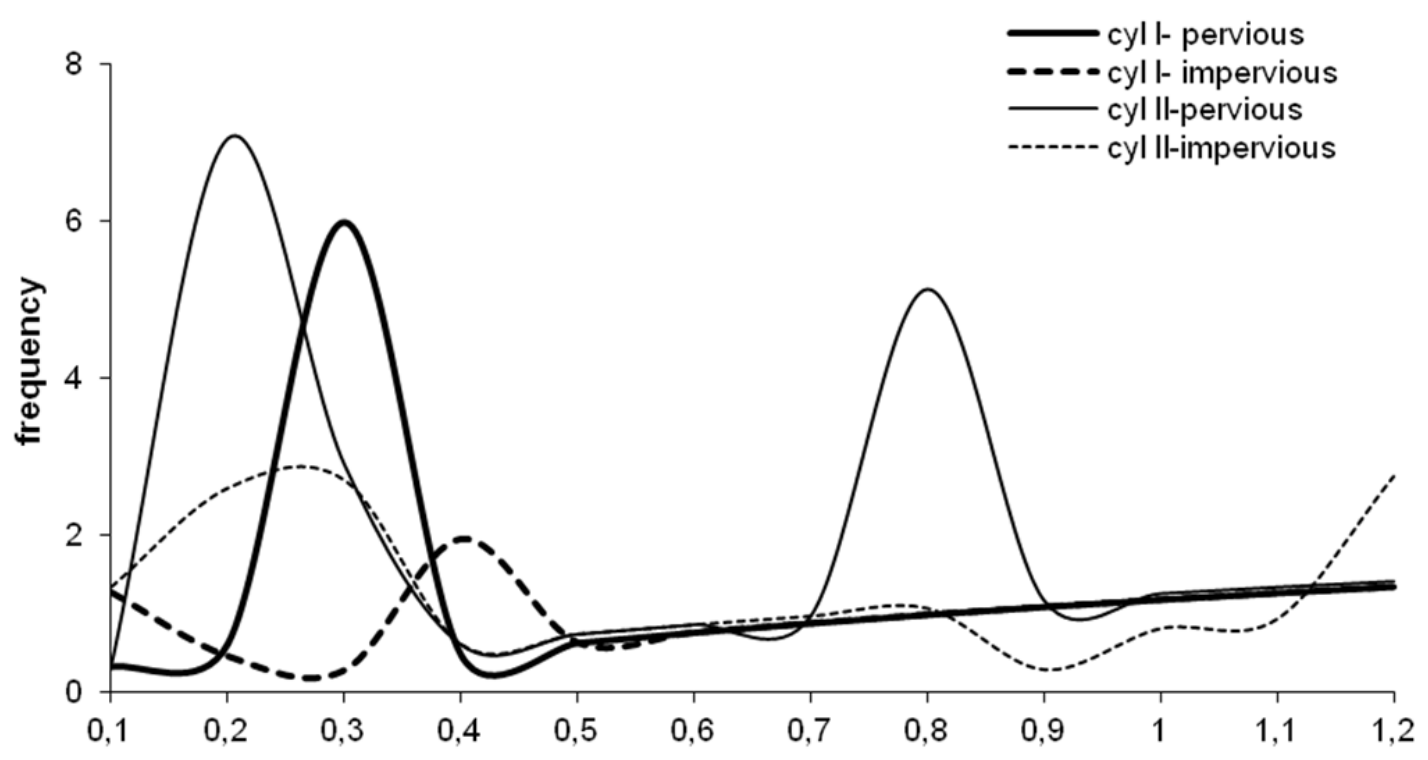

$\mathrm{h} / \mathrm{r} \mathbf{1}$

Fig.15. Frequency as a function of ratio of thickness to inner radius - poroelasti hollow cylinder - anti symmetric vibrations $(n=2)$.

The effect of fluid presence in a fluid-loaded poroelastic hollow cylinder surrounded by a fluid for the axially symmetric dilatational, flexural and anti-symmetric can be observed by comparing Figs 2-4 with Figs $13-15$, respectively. In the case of axially symmetric dilatational vibrations, the variation of frequency is more in the fluid-loaded poroelastic hollow cylinder surrounded by a fluid compared to the poroelastic hollow cylinder. Hence, the presence of internal and external fluids increases the variation of frequency of the poroelastic hollow cylinder in the case of axially symmetric dilatational vibrations. The frequency of the fluidloaded poroelastic hollow cylinder surrounded by a fluid is same as that of the poroelastic hollow cylinder beyond $h / \mathrm{r}_{1}=0.6$ in the case of flexural vibrations. Hence, the presence of internal and external fluids does not affect the frequency of the poroelastic hollow cylinder beyond $h / r_{l}=0.6$ in the case of flexural vibrations. In the case of anti symmetric vibrations, the frequency of the fluid-loaded poroelastic hollow cylinder surrounded by a fluid for a pervious surface is less than that of the poroelastic hollow cylinder for both cylinders I and II, whereas the phenomenon is reverse for an impervious surface. Thus, the presence of internal and external fluids decreases the frequency of the poroelastic hollow cylinder for a pervious surface and increases the frequency for an impervious surface for both cylinders I and II in the case of anti-symmetric vibrations.

On comparing Figs 5 and 13, it is observed that the frequency of the fluid-loaded poroelastic hollow cylinder is higher than that of the poroelastic hollow cylinder in the case of axially symmetric dilatational vibrations. Hence, the presence of an internal fluid increases the frequency of the poroelastic hollow cylinder in the case of axially symmetric vibrations. From Figs 6 and 14, it is clear that the frequency of the fluid-loaded poroelastic hollow cylinder is higher than that of the poroelastic hollow cylinder below $h / r_{I}=0.7$ and the frequencies are same for other values of $h / r_{l}$ in the case of flexural vibrations. Hence, the presence of the internal fluid does not affect the frequency of the poroelastic hollow cylinder beyond $h / r_{l}=0.7$ in the case of flexural vibrations. In the case of anti- symmetric vibrations, the frequency of the fluid-loaded poroelastic hollow cylinder for a pervious surface is less than that of the poroelastic hollow cylinder for both cylinders I and II as shown in Figs 7 and 15. Thus, the presence of the internal fluid decreases the frequency of the poroelastic hollow cylinder for a pervious surface for both cylinders I and II in the case of anti-symmetric vibrations.

From Figs 10 and 13, it is clear that the variation of frequency in the poroelastic cylinder surrounded by a fluid is more compared to the frequency of the poroelastic hollow cylinder below $h / \mathrm{r}_{1}=0.5$ and the frequencies are same for other values of $h / r_{l}$ in the case of axially symmetric dilatational vibrations. Hence, the presence of the external fluid does not affect the frequency of the poroelastic hollow cylinder beyond $h / r_{1}=0.5$ 
in the case of axial symmetric dilatational vibrations._From Figs 11 and 14, it is clear that the frequency of the poroelastic hollow cylinder surrounded by a fluid is same as that of the poroelastic hollow cylinder beyond $h / r_{I}$ $=0.6$ in the case of flexural vibrations. Hence, the presence of the internal fluid does not affect the frequency of the poroelastic hollow cylinder beyond $h / r_{l}=0.6$ in the case of flexural vibrations. In the case of anti- symmetric vibrations, the frequency of the poroelastic hollow cylinder surrounded by a fluid for a pervious surface is less than that of the poroelastic hollow cylinder for both cylinders I and II, whereas for an impervious surface a reverse phenomenon is observed as shown in Figs 12 and 15. Thus, the presence of the external fluid decreases the frequency of the poroelastic hollow cylinder for a pervious surface and increases the frequency for an impervious surface for both cylinders I and II in the case of anti-symmetric vibrations.

The effect of fluid presence in a fluid-loaded poroelastic bore for the cases of axially symmetric dilatational vibrations can be observed by comparing Figs 8 and 9, which show that the phase velocity of the fluid-loaded poroelastic bore is higher than that of the poroelastic empty bore for cylinder II. Thus, the presence of a fluid increases the phase velocity of the poroelastic bore II in the case of axially symmetric dilatational vibrations.

\section{Conclusion}

The analysis of vibrations of a fluid-loaded poroelastic hollow cylinder surrounded by a fluid has lead to the following conclusions:

(i) The shear and dilatational vibrations of the fluid-loaded poroelastic hollow cylinder surrounded by a fluid are uncoupled in the case of axially symmetric vibrations.

(ii) Axially symmetric shear vibrations are independent of the nature of the surface.

(iii) The variation of frequency in the fluid-loaded poroelastic hollow cylinder surrounded by a fluid is more in the case of anti-symmetric vibrations than axially symmetric dilatational and flexural vibrations.

(iv) In general, the frequency of the fluid-loaded poroelastic hollow cylinder is increasing in the case of axially symmetric dilatational vibrations.

(v) The frequency is steady and increasing for a moderately thick poroelastic cylinder in the case of flexural vibrations.

(vi) In general, the phase velocity of the fluid-loaded bore I for a pervious surface is decreasing.

(vii) In general, the phase velocity of bore II is less than that of bore I.

(viii) The frequency is steady and increasing for a moderately thick poroelastic hollow cylinder surrounded by a fluid in the case of axially symmetric dilatational and flexural vibrations.

(ix) In general, the variation of frequency of the poroelastic hollow cylinder surrounded by a fluid is more in the case of anti-symmetric vibrations compared to axially symmetric dilatational and flexural vibrations.

(x) The presence of both internal and external fluids increases the variation of frequency of a the poroelastic hollow cylinder in the case of axially symmetric dilatational vibrations.

(xi) The presence of both internal and external fluids decreases the variation of frequency of the poroelastic hollow cylinder in the case of anti-symmetric vibrations.

(xii) The presence of the internal fluid increases the frequency of the poroelastic hollow cylinder in the case of axially symmetric vibrations.

(xiii) The presence of the external fluid decreases the frequency of the poroelastic hollow cylinder for a pervious surface and increases the frequency for an impervious surface in the case of anti-symmetric vibrations.

(xiv) The presence of fluid increases the phase velocity of poroelastic bore II.

(xv) There is no effect of fluid presence on phase velocity of poroelastic bore I.

\section{Nomenclature}

$$
\begin{aligned}
A, N, Q, R & - \text { poroelastic constants } \\
b & - \text { dissipation coefficient } \\
C & - \text { phase velocity } \\
e & - \text { dilatation of solid }
\end{aligned}
$$




$$
\begin{aligned}
H_{n}^{(1)} & - \text { Hankel function of first kind of order } n \\
J_{n} & - \text { Bessel function of first kind of order } n \\
P_{f}^{(j)} & - \text { fluid pressure } \\
r_{1} & - \text { inner radius of cylinder } \\
r_{2} & - \text { outer radius of cylinder } \\
S & - \text { liquid pressure } \\
t & - \text { time } \\
\boldsymbol{U} & - \text { liquid displacement } \\
\boldsymbol{u} & - \text { solid displacement } \\
V_{1} & - \text { dilatational wave velocity of first kind } \\
V_{2} & - \text { dilatational wave velocity of second kind } \\
V_{3} & - \text { shear wave velocitiy } \\
V_{f}^{(j)} & - \text { velocity of sound in fluid } \\
Y_{n} & - \text { Bessel function of second kind of order } n \\
\varepsilon & - \text { dilatation of liquid } \\
\rho_{11}, \rho_{12}, \rho_{22} & - \text { mass coefficients } \\
\rho_{f}^{(j)} & - \text { density of the fluid } \\
\sigma_{k l} & - \text { stresses in poroelastic solid } \\
\omega & - \text { circular frequency } \\
\nabla^{2} & - \text { Laplacian operator }
\end{aligned}
$$

\section{References}

Abousleiman Y. and Cui L. (1998): Poroelastic solutions in transversely isotropic media for wellbore cylinders. International Journal of Solids and Structures, vol.35, pp.4905-4929.

Ahmed Shah S. (2008): Axially symmetric vibrations of fluid-filled poroelastic circular hollow cylinders. - Journal of Sound and Vibration, vol.318, pp.389-405.

Ahmed Shah S. and Tajuddin M. (2010): On flexural vibrations of poroelastic circular hollow cylinders immersed in an acoustic medium. - Special Topics and Reviews in Porous Media, vol.1, pp.67-78.

Biot M.A. (1956): The theory of propagation of elastic waves in fluid-saturated porous solid. - J. Acous. Soc. Am., vol.28, pp.168-178.

Cui L., Cheng A.H.D. and Abousleiman Y. (1997): Poroelastic solutional os an inclined borehole. - Transactions of ASME, Journal of Applied Mechanics, vol.64, pp.32-38.

Fatt I. (1959): The Biot-Willis elastic coefficients for a sandstone. - J. Appl. Mech., vol.26, pp.296-297.

Gazis D.C. (1958): Exact analysis of plane-strain vibrations in thick-walled hollow cylinders. - J. Acoust. Soc. Am., vol.30, pp.786-794.

Kumar Ram (1966): Axially symmetric vibrations of thin cylindrical elastic shell, filled with non-viscous fluid. Acustica, vol.17, pp.218-222.

Kumar Ram (1971): Flexural vibrations of fluid-filled circular hollow cylinders. - Acustica, vol.24, pp.137-146.

Malla Reddy P. and Tajuddin M. (2000): Exact analysis of plane-strain vibrations of thick-walled hollow poroelastic cylinders. - International Journal of Solids and Structures, vol.37, pp.3439-3456.

Malla Reddy P. and Tajuddin M. (2010): Axially symmetric vibrations of composite poroelastic cylinders in the context of fretting fatigue. - Special Topics and Reviews in Porous Media, vol.1, pp.311-320.

Marilyn J. Berliner and Solecki R. (1996): Wave propagation in fluid-loaded, transversely isotropic cylinders. Part I. Analytical formulation. - J. Acoust. Soc. Am., vol.99, pp.1841-1847. 
Nageswara Nath C., Tajuddin M. and Manoj Kumar J. (2011): On parametric model of loose bonding between poroelastic half-spaces. - Journal of Vibration and Control, vol.18, pp.1261-1274.

Plona T.J., Sinha B.K., Kostek S. and Chang S.K. (1992): Axisymmetric wave propagation in fluid-loaded cylindrical shells I: Theory versus experiment. - J. Acoust. Soc. Am., vol.92, pp.1144-1155.

Ram Kumar (1972): Dispersion of axially symmetric waves in empty and fluid-filled hollow cylinders. - Acustica, vol.27, pp.317-329.

Sharma M.D. and Gogna M.L. (1990): Propagation of elastic waves in a cylindrical bore in a liquid saturated porous solid. - Geophysical Journal International, vol.103, pp.47-54.

Tajuddin M., Nageswara Nath C. and Manoj Kumar J. (2011): Axial-shear vibrations of an infinitely long poroelastic composite circular cylinder. - Special Topics and Reviews in Porous Media, vol.2, pp.133-143.

Yew C.H. and Jogi P.N. (1976): Study of wave motions in fluid-saturated porous rocks. - J. Acoust. Soc. Am., vol.60, pp.2-8.

Received: November 19, 2011

Revised: August 4, 2012 